TRANSACTIONS OF THE

AMERICAN MATHEMATICAL SOCIETY

Volume 360, Number 9, September 2008, Pages 4863-4887

S 0002-9947(08)04508-X

Article electronically published on April 16, 2008

\title{
EQUIDISTRIBUTION AND INTEGRAL POINTS FOR DRINFELD MODULES
}

\author{
D. GHIOCA AND T. J. TUCKER
}

\begin{abstract}
We prove that the local height of a point on a Drinfeld module can be computed by averaging the logarithm of the distance to that point over the torsion points of the module. This gives rise to a Drinfeld module analog of a weak version of Siegel's integral points theorem over number fields and to an analog of a theorem of Schinzel's regarding the order of a point modulo certain primes.
\end{abstract}

\section{INTRODUCTION}

In 1929, Siegel ( Sie29] ) proved that if $C$ is an affine curve defined over a number field $K$ and $C$ has at least three points at infinity, then there are finitely many $K$-rational points with integral coordinates. The proof of this famous theorem uses diophantine approximation along with the fact that certain groups of rational points are finitely generated; when $C$ has genus greater than 0 , the group in question is the Mordell-Weil group of the Jacobian of $C$, while when $C$ has genus 0 , the group in question is the group of $S$-units in a finite extension of $K$.

When $C$ is a curve of genus 0 , it is simple to give a notion of integrality that is more flexible than the notion of integral coordinates. Let $S$ be a finite set of places of $K$. Viewing $\mathbb{P}^{1}(K)$ as $K$ plus a point at infinity, we say that $\beta \in K$ is $S$-integral with respect to $\alpha \in K$ if at each finite place $v$ of $K$ outside of $S$, we have $|\alpha-\beta|_{v} \geq 1$ and $\min \left(|\alpha|_{v},|\beta|_{v}\right) \leq 1$ (see Section 2.6 for a more precise definition of $S$-integrality). Similarly, we say that $\beta$ is $S$-integral with respect to the point at infinity if $|\beta|_{v} \leq 1$ for all points outside of $S$. Using these conventions, along with the convention of the usual Weil height $h(\alpha)$ of an algebraic number $\alpha$, Siegel's method of proof can be described very easily. The set of points $\beta$ that are integral with respect to any two points in $\mathbb{P}_{K}^{1}$ can be realized as a subset of the group $\Gamma$ of $S$-units in some extension $L$ of $K$. Since $\Gamma / m \Gamma$ is finitely generated, if $\alpha$ is another point (making a total of three) for which there are infinitely many $\beta_{i} \in \Gamma$ that are integral with respect to $\alpha$, then there is some $\gamma \in \Gamma$ such that infinitely many $\gamma+\beta_{i}$ have $m$-th roots $\beta_{i}^{\prime}$ in $L$. The fact that the $\beta_{i}$ are $S$-integral with respect to $\alpha$ means that $\sum_{v \in S}-\log \left|\beta_{i}-\alpha\right|_{v}=h(\beta)+O(1)$ for all $\beta$. Then there is an $m$-th

Received by the editors September 5, 2006.

2000 Mathematics Subject Classification. Primary 11G50; Secondary 11J68, 37F10.

Key words and phrases. Drinfeld module, heights, Diophantine approximation.

(C)2008 American Mathematical Society Reverts to public domain 28 years from publication 
root $\alpha^{\prime}$ of $\alpha+\gamma$, and infinitely many $m$-th roots $\beta_{i}^{\prime} \in L$ of $\beta_{i}+\gamma$ such that

$$
\begin{aligned}
\sum_{v \in S}-\log \left|\beta_{i}^{\prime}-\alpha^{\prime}\right|_{v}=\sum_{v \in S}-\log \left|\beta_{i}-\alpha\right|_{v}+O(1) & =h\left(\beta_{i}\right)+O(1) \\
& =m h\left(\beta_{i}^{\prime}\right)+O(1),
\end{aligned}
$$

which violates a theorem from diophantine approximation, such as Roth's theorem ( Rot55]) or Siegel's own result, also found in [Sie29].

Now, suppose that the base field of our curves is a function field $K$ over a finite field. When $C$ is a nonisotrivial curve of positive genus, Siegel's theorem is still true (see [Vol90] and [Vol91]), but Siegel's theorem is not true for the projective line with three points removed. For example, let $K$ be any function field over a finite field (of characteristic $p$ ) and let $x$ be any point in $K$ that is not in the constant field. Let $S$ be the set of places for which either $|x|_{v} \neq 1$ or $|x-1|_{v} \neq 1$. Then any power of $x$ is $S$-integral with respect to both 0 and $\infty$ and, since $\left|x^{p^{m}}-1\right|_{v}=$ $\left|(x-1)^{p^{m}}\right|_{v}=|x-1|_{v}^{p^{m}}$ for any positive integer $m$, we see that $x^{p^{m}}$ is $S$-integral with respect to 1 as well. Note that the diophantine approximation results over function fields are much weaker than Roth's (or Siegel's or Dyson's) theorem (see Vol97, or Osg75, for example).

In this paper we will prove an analog of Siegel's theorem for cyclic $\phi$-submodules of $\mathbb{G}_{a}(K)$ (under the action of a Drinfeld module $\phi$ ). In some sense this is surprising in light of the counterexample above for $\mathbb{Z}$-submodules of $\mathbb{G}_{m}(K)$. On the other hand, there are many other famous theorems over number fields that have analogs in characteristic $p$ in the context of Drinfeld modules. For example, Ghioca Ghi05] and Scanlon Sca02 proved Mordell-Lang and Manin-Mumford statements, respectively, for Drinfeld modules; these had both been conjectured by Denis (Den92a ). Bosser formulated and proved (Bos00]) a variant of the Bogomolov conjecture for Drinfeld modules (unpublished, but the main ingredient of his result is contained in [Bos02]). Ghioca (Ghi06) proved a Drinfeld module analog of Szpiro, Ullmo, and Zhang's equidistribution theorem ([SUZ97]) for abelian varieties. In this paper we explore further aspects of equidistribution in the context of Drinfeld modules and demonstrate a connection between equidistribution and integrality of points.

We will prove the following theorem.

Theorem 1.1. Let $K$ be a finite extension of $\mathbb{F}_{q}(t)$. Let $\phi: \mathbb{F}_{q}[t] \rightarrow K\{\tau\}$ be a Drinfeld module and let $S$ be a finite set of places of $K$. If $\beta \in K$ is not torsion for $\phi$, then there are finitely many $Q \in \mathbb{F}_{q}[t]$ such that $\phi_{Q}(\beta)$ is $S$-integral for the point 0.

We will derive Theorem 1.1 as a consequence of Theorem 3.1, which states that the local height of a point $\beta$ can be computed by averaging the function $\log |x-\beta|_{v}$ over the periodic points of $\phi$. Theorem 3.1 can be regarded as an equidistribution theorem, since local canonical heights can typically be computed by integrating $\log |x-\beta|_{v}$ against an invariant measure (see Section 5 for more details). Theorem 3.1 also gives rise to Theorem 4.1, which is a result about the order of the reduction of a point modulo various primes. Determining the order of the reduction of a nontorsion point at various primes was also studied in the context of abelian varieties (see Pin04).

Let us now give a brief outline of the paper. We begin with some preliminaries about canonical heights on Drinfeld modules in Section 2, Following that we prove Theorem 3.1. The main ingredient for Theorem 3.1 is Corollary 3.13 which provides 
a new way of defining local canonical heights for a Drinfeld module. For the places lying over the place at infinity for the Drinfeld module (see Section 2 for a more precise definition of these places), this is proved using linear forms in logarithms (as in [Bos99]); for the "finite" places, this is proved using an elementary method described in Lemmas 3.10 and 3.12. Theorem 1.1 follows easily from Theorem 3.1. The proof of Theorem 4.1 requires a somewhat lengthy counting argument, which is detailed in Section 4. In Section [5] we discuss further generalizations, including analogs of work of Silverman ([Sil93]), Baker/Ih/Rumely (BIR05]), and Szpiro/Tucker ([ST] $)$. The most general question we pose may be viewed as a possible Siegel's integral points theorem for arbitrary finitely generated $\phi$-submodules of $\mathbb{G}_{a}(\bar{K})$. In a recent paper [GT07] we answer positively our question under one mild technical hypothesis and also assuming a natural conjecture for linear forms in logarithms at finite places for a Drinfeld module (which is the analog of Bosser's result [Bos99] for the linear forms in logarithms at infinite places).

\section{Notation}

2.1. Drinfeld modules. We begin by defining a Drinfeld module. Let $p$ be a prime and let $q$ be a power of $p$. Let $A:=\mathbb{F}_{q}[t]$, let $K$ be a finite extension of $\mathbb{F}_{q}(t)$, and let $\bar{K}$ be an algebraic closure of $K$. We let $\tau$ be the Frobenius on $\mathbb{F}_{q}$, and we extend its action on $\bar{K}$. Let $K\{\tau\}$ be the ring of polynomials in $\tau$ with coefficients from $K$ (the addition is the usual addition, while the multiplication is the composition of functions).

A Drinfeld module is a morphism $\phi: A \rightarrow K\{\tau\}$ for which the coefficient of $\tau^{0}$ in $\phi(a)=: \phi_{a}$ is $a$ for every $a \in A$, and there exists $a \in A$ such that $\phi_{a} \neq a \tau^{0}$. The definition given here represents what Goss Gos96 calls a Drinfeld module of "generic characteristic".

We note that usually, in the definition of a Drinfeld module, $A$ is the ring of functions defined on a projective nonsingular curve $C$, regular away from a closed point $\eta \in C$. For our definition of a Drinfeld module, $C=\mathbb{P}_{\mathbb{F}_{q}}^{1}$ and $\eta$ is the usual point at infinity on $\mathbb{P}^{1}$. On the other hand, every ring of regular functions $A$ as above contains $\mathbb{F}_{q}[t]$ as a subring, where $t$ is a nonconstant function in $A$.

A point $\alpha$ is torsion for the Drinfeld module action if and only if there exists $Q \in A \backslash \mathbb{F}_{q}$ such that $\phi_{Q}(\alpha)=0$. The monic polynomial $Q$ of minimal degree which satisfies $\phi_{Q}(\alpha)=0$ is called the order of $\alpha$. Since each polynomial $\phi_{Q}$ is separable, the torsion submodule $\phi_{\text {tor }}$ lies in the separable closure $K^{\text {sep }}$ of $K$.

For every field extension $K \subset L$, the Drinfeld module $\phi$ induces an action on $\mathbb{G}_{a}(L)$ by $a * x:=\phi_{a}(x)$, for each $a \in A$. We call $\phi$-submodules subgroups of $\mathbb{G}_{a}(\bar{K})$ which are invariant under the action of $\phi$. As shown in Poo95, $\mathbb{G}_{a}(K)$ is a direct sum of a finite torsion $\phi$-submodule with a free $\phi$-submodule of rank $\aleph_{0}$.

We will often use sums of the form $\sum_{P \mid Q}$ over divisors $P \in A$ of a fixed polynomial $Q \in A$. These sums will always be taken over the monic divisors $P$ of $Q$.

2.2. Valuations and Weil heights. Let $M_{\mathbb{F}_{q}(t)}$ be the set of places on $\mathbb{F}_{q}(t)$. We denote by $v_{\infty}$ the place in $M_{\mathbb{F}_{q}(t)}$ such that $v_{\infty}\left(\frac{f}{g}\right)=\operatorname{deg}(g)-\operatorname{deg}(f)$ for every nonzero $f, g \in A=\mathbb{F}_{q}[t]$. We let $M_{K}$ be the set of valuations on $K$. Then $M_{K}$ is a 
set of valuations which satisfies a product formula (see [Ser97, Chapter 2]). Thus

- for each nonzero $x \in K$, there are finitely many $v \in M_{K}$ such that $|x|_{v} \neq 1$; and

- for each nonzero $x \in K$, we have $\prod_{v \in M_{K}}|x|_{v}=1$.

We may use these valuations to define a Weil height for each $x \in K$ as

$$
h(x)=\sum_{v \in M_{K}} \log \max \left(|x|_{v}, 1\right) .
$$

We may also extend the Weil height to all of $\bar{K}$ in a coherent way (see Chapter 2 of [Ser97] for a detailed discussion of the construction of heights on $\bar{K}$ ). More specifically, if $L$ is an extension of $K$, then we can define a set $M_{L}$ of absolute values $|\cdot|_{w}$ on $L$ such that

$$
|y|_{v}=\prod_{\substack{w \mid v \\ w \in M_{L}}}|y|_{w} .
$$

Note that we write $w \mid v$ when $|\cdot|_{w}$ restricts to some power of $|\cdot|_{v}$ on $K$, but that $|\cdot|_{w}$ is not in general equal to $|\cdot|_{v}$ on $K$ (see [Ser97, pages 9-11]). We use these $|\cdot|_{w}$ to define a height for any $y$ in a finite extension $L$ of $K$ by

$$
h(y)=\sum_{w \in M_{L}} \log \max \left(|y|_{w}, 1\right) .
$$

It follows from (2.0.2), that for any $x \in K$ and any extension $L$ of $K$, we have

$$
\sum_{v \in M_{K}} \log \max \left(|x|_{v}, 1\right)=\sum_{w \in M_{L}} \log \max \left(|x|_{w}, 1\right) .
$$

Thus the height on all of $\bar{K}$ restricts to the original Weil height (2.0.1) on $K$. More generally, the definition of $h(y)$ in (2.0.3) does not depend on our choice of the field $L$ containing $y$. For more details, we refer the reader to [Ser97, Chapter 2].

Definition 2.1. Let $L$ be a finite extension of $K$. Each place in $M_{L}$ that lies over $v_{\infty}$ is called an infinite place. Each place in $M_{L}$ that does not lie over $v_{\infty}$ is called a finite place.

2.3. Canonical heights. Let $\phi: A \rightarrow K\{\tau\}$ be a Drinfeld module of rank $d$ (i.e. the degree of $\phi_{t}$ as a polynomial in $\tau$ equals $d$ ). The canonical height of $\beta \in \bar{K}$ relative to $\phi$ (see Den92b]) is defined as

$$
\widehat{h}(\beta)=\lim _{n \rightarrow \infty} \frac{h\left(\phi_{t^{n}}(\beta)\right)}{q^{n d}} .
$$

Denis Den92b showed that $\alpha$ is a torsion point for $\phi$ if and only if $\widehat{h}(\alpha)=0$.

For every finite extension $L$ of $K$, and for every $w \in M_{L}$, we let the local canonical height of $\beta \in L$ at $w$ be

$$
\widehat{h}_{w}(\beta)=\lim _{n \rightarrow \infty} \frac{\log \max \left(\left|\phi_{t^{n}}(\beta)\right|_{w}, 1\right)}{q^{n d}} .
$$

It is clear that $\sum_{w \in M_{L}} \widehat{h}_{w}(\beta)=\widehat{h}(\beta)$.

We will use the notion of Galois conjugate for points $\beta \in \bar{K}$. Even though this notion is classical, for the sake of completeness, we define it here. If $\sigma: \bar{K} \rightarrow \bar{K}$ is a field automorphism fixing $K$, then $\beta^{\sigma}:=\sigma(\beta)$ is called a Galois conjugate of $\beta$. 
2.4. Completions and filled Julia sets. By abuse of notation, we let $\infty \in M_{K}$ denote any place extending the place $v_{\infty}$. We let $K_{\infty}$ be the completion of $K$ with respect to $|\cdot|_{\infty}$. We let $\overline{K_{\infty}}$ be an algebraic closure of $K_{\infty}$. We let $\mathbb{C}_{\infty}$ be the completion of $\overline{K_{\infty}}$. Then $\mathbb{C}_{\infty}$ is a complete, algebraically closed field. Note that $\mathbb{C}_{\infty}$ depends on our choice for $\infty \in M_{K}$ extending $v_{\infty}$. However, each time we will work with only one such place $\infty$, and so there will be no possibility of confusion.

Next, we define the $w$-adic filled Julia set $J_{\phi, w}$ corresponding to the Drinfeld module $\phi$ and to each place $w$ of $M_{L}$ (where $L$ is any finite extension of $K$ ). Let $\mathbb{C}_{w}$ be the completion of an algebraic closure of $L_{w}$. Then $|\cdot|_{w}$ extends to a unique absolute value on all of $\mathbb{C}_{w}$. The set $J_{\phi, w}$ consists of all $x \in \mathbb{C}_{w}$ for which $\left\{\left|\phi_{Q}(x)\right|_{w}\right\}_{Q \in A}$ is bounded. It is immediate to see that $x \in J_{\phi, w}$ if and only if $\left\{\left|\phi_{t^{n}}(x)\right|_{w}\right\}_{n \geq 1}$ is bounded.

One final note on absolute values: as noted above, each place $v \in M_{K}$ extends to a unique absolute value $|\cdot|_{v}$ on all of $\mathbb{C}_{v}$. We fix an embedding of $i: \bar{K} \longrightarrow \mathbb{C}_{v}$. For $x \in \bar{K}$, we denote $|i(x)|_{v}$ simply as $|x|_{v}$, by abuse of notation.

2.5. The coefficients of $\phi_{t}$. Each Drinfeld module is isomorphic to a Drinfeld module for which all the coefficients of $\phi_{t}$ are integral at all the places in $M_{K}$ which do not lie over $v_{\infty}$. Indeed, we let $B \in \mathbb{F}_{q}[t]$ be a product of all (the finitely many) irreducible polynomials $P \in \mathbb{F}_{q}[t]$ with the property that there exists a place $v \in M_{K}$ which lies over the place $(P) \in M_{\mathbb{F}_{q}(t)}$, and there exists a coefficient of $\phi_{t}$ which is not integral at $v$. Let $\gamma$ be a sufficiently large power of $B$. Then $\psi: A \rightarrow K\{\tau\}$ defined by $\psi_{Q}:=\gamma^{-1} \phi_{Q} \gamma$ (for each $Q \in A$ ) is a Drinfeld module isomorphic to $\phi$, and all the coefficients of $\psi_{t}$ are integral away from the places lying above $v_{\infty}$. Hence, from now on, we assume that all the coefficients of $\phi_{t}$ are integral away from the places lying over $v_{\infty}$. It follows that for every $Q \in A$, all coefficients of $\phi_{Q}$ are integral away from the places lying over $v_{\infty}$.

\subsection{Integrality and reduction.}

Definition 2.2. For a finite set of places $S \subset M_{K}$ and $\alpha \in \bar{K}$, we say that $\beta \in \bar{K}$ is $S$-integral with respect to $\alpha$ if for every place $v \notin S$, and for every pair of morphisms $\sigma, \tau: \bar{K} \rightarrow \bar{K}$, the following are true:

- if $\left|\alpha^{\tau}\right|_{v} \leq 1$, then $\left|\alpha^{\tau}-\beta^{\sigma}\right|_{v} \geq 1$.

- if $\left|\alpha^{\tau}\right|_{v}>1$, then $\left|\beta^{\sigma}\right|_{v} \leq 1$.

For more details about the definition of $S$-integrality, we refer the reader to [BIR05.

We also introduce the notation $\bar{\beta}$ for the reduction at a place $w \in M_{L}$ of a point $\beta \in L$, which is integral at $w$. When $\phi$ has good reduction at $w$ (i.e. for each nonzero $Q \in \mathbb{F}_{q}[t]$, all coefficients of $\phi_{Q}$ are $w$-adic integers, and the leading coefficient of $\phi_{Q}$ is a $w$-adic unit), we denote by $\bar{\phi}$ the reduction of $\phi$ at $w$. Note that when $\phi$ has good reduction, $\bar{\phi}$ is a well-defined Drinfeld module on $\mathbb{G}_{a}\left(k_{w}\right)$ of the same rank as $\phi$, where $k_{w}$ is the residue field at $w$. When $\phi$ does not have good reduction at $w$, we say that it has bad reduction at $w$. We observe that each place lying above $v_{\infty}$ is a place of bad reduction for $\phi$. 4.13).

The following fact is also proved in an equivalent form in Ghi07a] (see Lemma

Fact 2.3. Let $w$ be a place of good reduction for $\phi$, and let $\alpha \in \mathbb{C}_{w}$ be a torsion point for $\phi$. Then $\alpha$ is a $w$-adic integer. 
Proof. Assume $\alpha$ is not a $w$-adic integer. Let $Q \in \mathbb{F}_{q}[t]$ be the order of $\alpha$. Assume $\phi_{Q}=\sum_{j=0}^{l} b_{j} \tau^{j}$. Then for every $0 \leq j<l$,

$$
\left|b_{l} \alpha^{q^{l}}\right|_{w}=\left|\alpha^{q^{l}}\right|_{w}>\left|\alpha^{q^{j}}\right|_{w} \geq\left|b_{j} \alpha^{q^{j}}\right|_{w} .
$$

Hence $\left|\phi_{Q}(\alpha)\right|_{w}=\left|\alpha^{q^{l}}\right|_{w}>1$, which contradicts $\phi_{Q}(\alpha)=0$.

\section{Proofs of the MAIN THEOREMS}

We continue with the notation as in Section 2 The main goal of this section is to prove the following theorem. Then Theorem 1.1 will follow easily as a corollary.

Theorem 3.1. For any nontorsion $\beta \in \bar{K}$ and any nonzero irreducible $F \in K[Z]$ such that $F(\beta)=0$, we have

$$
(\operatorname{deg} F) \widehat{h}(\beta)=\sum_{v \in M_{K}} \lim _{Q \rightarrow \infty} \frac{1}{q^{d \operatorname{deg} Q}} \sum_{\phi_{Q}(y)=0} \log |F(y)|_{v} .
$$

Later (see Theorem 3.14), we will prove a similar result as in Theorem 3.1 valid also for torsion points $\beta$. Our proof of Theorem 3.1 will go through a series of lemmas and propositions which ultimately yield a new way to compute local heights (see Corollary 3.13). We will use in our argument a proposition proved by Bosser (see Théorème 1.1 of [Bos99]) as well as a lemma due to Taguchi (see Lemma (4.2) of [Tag93]).

Proposition 3.2 (Bosser). Let $\infty$ be a place lying over $v_{\infty}$. Let $\exp _{\phi}$ be the exponential map associated to $\phi$ at $\infty$ (see Gos96]). Let $y_{1}, \ldots, y_{n}$ be elements of $\mathbb{C}_{\infty}$ such that $\exp _{\phi}\left(y_{i}\right) \in \mathbb{G}_{a}(\bar{K})$. Then there exists a (negative) constant $C_{1}$ (depending on $\phi, y_{1}, \ldots, y_{n}$ ) such that for any $P_{1}, \ldots, P_{n} \in \mathbb{F}_{q}[t]$, either $P_{1} y_{1}+$ $\cdots+P_{n} y_{n}=0$ or

$$
\log \left|P_{1} y_{1}+\cdots+P_{n} y_{n}\right|_{\infty} \geq C_{1} \max _{1 \leq i \leq n}\left(\operatorname{deg}\left(P_{i}\right) \log \operatorname{deg}\left(P_{i}\right)\right) .
$$

Remark 3.3. In the translation of Théorème 1.1 of [Bos99] to our Proposition 3.2, we use the fact that for each polynomial $P \in \mathbb{F}_{q}[t]$, its height is its degree.

Lemma 3.4 (Taguchi). Let $\exp _{\phi}$ be the associated exponential map to $\phi$ with respect to a fixed place $\infty$ lying over $v_{\infty}$. Let $\mathcal{L}$ be the lattice of all elements $u \in \mathbb{C}_{\infty}$ such that $\exp _{\phi}(u)=0$. There exists an A-basis $\omega_{1}, \ldots, \omega_{d}$ for $\mathcal{L}$ such that for any polynomials $P_{1}, \ldots, P_{d} \in \mathbb{F}_{q}[t]$, we have

$$
\left|P_{1} \omega_{1}+\cdots+P_{d} \omega_{d}\right|_{\infty}=\max _{1 \leq i \leq d}\left|P_{i} \omega_{i}\right|_{\infty} .
$$

Remark 3.5. The elements $\omega_{i}$ from Lemma 3.4 form a basis for $\mathcal{L}$ of "successive minima" (as defined in Tag93).

The following result appears in a similar form in Gos96 (see Proposition 4.14.2).

Lemma 3.6. With the notation as in Lemma 3.4, there exists a constant $C_{2}>0$ such that $\exp _{\phi}$ induces an isomorphism from the metric space $B_{1}=\left\{z \in \mathbb{G}_{a}\left(\mathbb{C}_{\infty}\right) \mid\right.$ $\left.|z|_{\infty} \leq C_{2}\right\}$ to itself.

Proof. As shown in Gos96,

$$
\exp _{\phi}(z)=z \prod_{\substack{\lambda \in \mathcal{L} \\ \lambda \neq 0}}\left(1-\frac{z}{\lambda}\right) .
$$


Because $\mathcal{L}$ is discrete in $|\cdot|_{\infty}$, there exists $C_{2}>0$ such that for every nonzero $\lambda \in \mathcal{L}$, we have $|\lambda|_{\infty}>C_{2}$. Thus, for each such $\lambda$, if $|z|_{\infty} \leq C_{2}$, then $\left|1-\frac{z}{\lambda}\right|_{\infty}=1$. Therefore, the ball

$$
B_{1}:=\left\{\left.z \in \mathbb{G}_{a}\left(\mathbb{C}_{\infty}\right)|| z\right|_{\infty} \leq C_{2}\right\}
$$

is mapped by $\exp _{\phi}$ into itself (preserving the distance). Moreover, $\exp _{\phi}$ is injective on $B_{1}$ because $\mathcal{L} \cap B_{1}=\{0\}$. Finally, $\exp _{\phi}: B_{1} \rightarrow B_{1}$ is surjective as can easily be seen by considering the entire function $f_{\alpha}(z):=\exp _{\phi}(z)-\alpha$ (for each $\alpha \in B_{1}$ ). Then $f_{\alpha}$ has a zero on $B_{1}$ (see the first slope of its associated Newton polygon).

Proposition 3.7. Let $L$ be a finite extension of $K$, and let $\infty \in M_{L}$ be a place lying above $v_{\infty}$. Let $\beta \in L$ be any nontorsion point for the Drinfeld module $\phi$ that is in $J_{\phi, \infty}$. Then

$$
\lim _{\operatorname{deg} Q \rightarrow \infty} \frac{\log \left|\phi_{Q}(\beta)\right|_{\infty}}{q^{d \operatorname{deg} Q}}=0 .
$$

Proof. Let $\epsilon$ be any real number greater than zero. Since $\beta$ is in the filled Julia set of $\phi$, we know that $\left|\phi_{Q}(\beta)\right|_{\infty}$ is bounded, so

$$
\frac{\log \left|\phi_{Q}(\beta)\right|_{\infty}}{q^{d \operatorname{deg} Q}}<\epsilon
$$

when $\operatorname{deg} Q$ is sufficiently large.

Now, if $\frac{\log \left|\phi_{Q}(\beta)\right|_{\infty}}{q^{d \operatorname{deg} Q}}<-\epsilon$ for $\operatorname{deg}(Q)$ sufficiently large, then we have $\left|\phi_{Q}(\beta)\right|_{\infty}<$ $C_{2}$ where $C_{2}$ is as in Lemma 3.6. We will use Proposition 3.2 to derive a contradiction. First, we let $u \in \mathbb{C}_{\infty}$ such that $\exp _{\phi}(u)=\beta$. We also choose a basis $\omega_{1}, \ldots, \omega_{d}$ of "successive minima" as in Lemma 3.4. Throughout our argument, $u, \omega_{1}, \ldots, \omega_{d}$ are fixed.

Because $\left|\phi_{Q}(\beta)\right|_{\infty}<C_{2}$, there exists $y$ such that $|y|_{\infty}=\left|\phi_{Q}(\beta)\right|_{\infty}$ and $\exp _{\phi}(y)=$ $\phi_{Q}(\beta)$. Since $Q u$ satisfies $\exp _{\phi}(Q u)=\phi_{Q}(\beta)$, there exist $P_{1}, \ldots, P_{d} \in A$ such that

$$
y=Q u+P_{1} \omega_{1}+\cdots+P_{d} \omega_{d} .
$$

Moreover, because of Lemma 3.6, there exists no $y^{\prime}$ with

$$
\left|y^{\prime}\right|_{\infty}<\left|\phi_{Q}(\beta)\right|_{\infty}<C_{2}
$$

such that $\exp _{\phi}\left(y^{\prime}\right)=\phi_{Q}(\beta)$. Thus, $|Q u|_{\infty} \geq|y|_{\infty}$. Therefore, we have

$$
\left|P_{1} \omega_{1}+\cdots+P_{d} \omega_{d}\right|_{\infty} \leq|Q u|_{\infty}
$$

(because otherwise $|y|_{\infty}>|Q u|_{\infty}$ ).

Thus, by Lemma 3.4 we see that

$$
\max _{i}\left|P_{i} \omega_{i}\right|_{\infty} \leq|Q|_{\infty} \cdot|u|_{\infty}
$$

Taking logarithms in (3.7.1), we obtain

$$
\max _{i} \operatorname{deg}\left(P_{i}\right) \leq \operatorname{deg}(Q)+\log |u|_{\infty}-\min _{i} \log \left|\omega_{i}\right|_{\infty} .
$$

Let $C_{3}:=\log |u|_{\infty}$. Hence, $\max _{i} \operatorname{deg}\left(P_{i}\right) \leq \operatorname{deg} Q+C_{4}$ (the constant $C_{4}$ depends only on $C_{3}$ and $\phi$, as the $\left(\omega_{i}\right)_{i=1}^{d}$ is a fixed basis of successive minima for the corresponding lattice of $\phi$ at $\infty$ ). Thus, Proposition 3.2 gives

$$
\begin{aligned}
\log \left|\phi_{Q}(\beta)\right|_{\infty}=\log |y|_{\infty} & =\log \left|Q u+P_{1} \omega_{1}+\cdots+P_{d} \omega_{d}\right|_{\infty} \\
& \geq C_{1}\left(\operatorname{deg} Q+C_{4}\right) \log \left(\operatorname{deg} Q+C_{4}\right) .
\end{aligned}
$$


Note that we know $y \neq 0$, because $\phi_{Q}(\beta) \neq 0$ (and hence, we can apply the inequality from Proposition 3.2$)$. For large $Q$, this means that $\frac{\log \left|\phi_{Q}(\beta)\right|_{\infty}}{q^{d \operatorname{deg} Q}} \geq-\epsilon$, which completes our proof.

Corollary 3.8. For any nontorsion $\beta \in \bar{K}$ and for any place $\infty \in M_{K(\beta)}$ lying above $v_{\infty}$, we have

$$
\lim _{\operatorname{deg} Q \rightarrow \infty} \frac{\log \left|\phi_{Q}(\beta)\right|_{\infty}}{q^{d \operatorname{deg} Q}}=\widehat{h}_{\infty}(\beta) .
$$

Proof. If $\beta$ is in the filled Julia set, then this is Proposition 3.7. If $\beta$ is not in the filled Julia set, then for large $n$, we have

$$
\left|\phi_{t^{n}}(\beta)\right|_{\infty}>\max \left(\left|\phi_{t^{m}}(\beta)\right|_{\infty}, 1\right)
$$

for all $m<n$. So, for $Q$ of large degree we have

$$
\left|\phi_{Q}(\beta)\right|_{\infty}=\left|\phi_{t^{\operatorname{deg} Q}}(\beta)\right|_{\infty}=\max \left(\left|\phi_{t^{\operatorname{deg} Q}}(\beta)\right|_{\infty}, 1\right) .
$$

Our claim then follows from the definition of the local canonical height with respect to $\phi$.

Let $L$ be a finite extension of $K$ containing $\beta$. Now we deal with the places $w$ of $L$ which do not lie over $v_{\infty}$. We recall that for such $w$, all coefficients of $\phi_{Q}$ are integral at $w$ for each $Q \in A$.

If $\beta \notin J_{\phi, w}$, then $\left|\phi_{Q}(\beta)\right|_{w}$ is unbounded as $\operatorname{deg} Q \rightarrow \infty$. Then, by the definition of the local canonical height (see also the proof of Corollary 3.8), we have

$$
\widehat{h}_{w}(\beta)=\lim _{\operatorname{deg}(Q) \rightarrow \infty} \frac{\log \left|\phi_{Q}(\beta)\right|_{w}}{q^{d \operatorname{deg}(Q)}} .
$$

We will show that when $\beta \in J_{\phi, w}$, the limit above is equal to 0 .

Proposition 3.9. Let $L$ be a finite extension of $K$, and let $w \in M_{L}$ be a place not lying over $v_{\infty}$. Let $\beta \in L$ be a nontorsion point which is also in $J_{\phi, w}$. Then

$$
\lim _{\operatorname{deg}(Q) \rightarrow \infty} \frac{\log \left|\phi_{Q}(\beta)\right|_{w}}{q^{d \operatorname{deg}(Q)}}=0 .
$$

Proof. Because $\beta$ is in the $w$-adic filled Julia set, $\left|\phi_{Q}(\beta)\right|_{w}$ is bounded. Hence, for every $\epsilon>0$, there exists an integer $N_{\epsilon}^{+}$such that if $\operatorname{deg}(Q)>N_{\epsilon}^{+}$, then

$$
\frac{\log \left|\phi_{Q}(\beta)\right|_{w}}{q^{d \operatorname{deg}(Q)}}<\epsilon
$$

It remains to show that for every $\epsilon>0$ there exists an integer $N_{\epsilon}^{-}$such that if $\operatorname{deg}(Q)>N_{\epsilon}^{-}$, then

$$
\frac{\log \left|\phi_{Q}(\beta)\right|_{w}}{q^{d \operatorname{deg}(Q)}}>-\epsilon
$$

For proving (3.9.1), we start with a general lemma about valuations.

Lemma 3.10. Let $F(X)=b_{n} X^{n}+\cdots+b_{1} X$ be a polynomial such that $\left|b_{i}\right|_{w} \leq 1$ for all $i$. Then $|F(x)|_{w}=\left|b_{1} x\right|_{w}$ whenever $|x|_{w}<\left|b_{1}\right|_{w}$. In particular, $|F(x)|_{w} \leq|x|_{w}$. 
Proof of Lemma 3.10. For any $i \geq 2$, we have

$$
\left|b_{i} x^{i}\right|_{w} \leq\left|b_{i} x^{2}\right|_{w}<\left|b_{i} b_{1} x\right|_{w} \leq\left|b_{1} x\right|_{w} .
$$

Applying the ultrametric inequality thus yields

$$
|F(x)|_{w}=\left|b_{n} x^{n}+\cdots+b_{1} x\right|_{w}=\left|b_{1} x\right|_{w} .
$$

The following result is an immediate corollary of the fact that all coefficients of $\phi_{Q}($ for $Q \in A)$ are $w$-adic integers.

Corollary 3.11. For $0<\epsilon \leq 1$, the set of polynomials $Q \in A$ such that $\left|\phi_{Q}(\beta)\right|_{w}<$ $\epsilon$ is an ideal in $A$.

We continue the proof of Proposition 3.9. The place $w$ restricts to a place on $\mathbb{F}_{q}(t)$ which corresponds to an irreducible polynomial $P \in A$.

Hence, for each polynomial $Q \in A$, we have $|Q|_{w}=|P|_{w}^{\operatorname{ord}_{P}(Q)}$, where $P^{\operatorname{ord}_{P}(Q)}$ is the largest power of $P$ dividing $Q$. The following lemma will finish the proof of Proposition 3.9 .

Lemma 3.12. There exists a positive constant $C_{w}$ such that

$$
\left|\phi_{Q}(\beta)\right|_{w} \geq C_{w}|P|_{w}^{\operatorname{ord}_{P}(Q)}
$$

for all $Q \in A$.

Proof. If there is no $F \in A$ such that $\left|\phi_{F}(\beta)\right|_{w}<|P|_{w}$, then we are done. Otherwise, let $G \in A$ generate the ideal of all polynomials $F$ such that $\left|\phi_{F}(\beta)\right|_{w}<$ $|P|_{w}<1$, and let $C_{w}:=\left|\phi_{G}(\beta)\right|_{w}$ (note that $\phi_{G}(\beta) \neq 0$ because $\beta$ is nontorsion).

Let $Q$ be any element in the ideal generated by $G$. We may write $Q=F G$ for a polynomial $F \in \mathbb{F}_{q}[t]$. We expand $F$ out $P$-adically, i.e. we write

$$
F=R_{n} P^{n}+\cdots+R_{1} P+R_{0}
$$

where each $R_{i}$ is a polynomial of degree less than $\operatorname{deg} P$. Since $\left|R_{i}\right|_{w}=1$ for each $i$, we have

$$
\left|\left(\phi_{R_{i}} \phi_{G}\right)(\beta)\right|_{w}=C_{w}<|P|_{w}
$$

by Lemma 3.10. Moreover, also by Lemma 3.10, we have

$$
\left|\left(\phi_{P} \phi_{R_{i}} \phi_{G}\right)(\beta)\right|_{w}=C_{w}|P|_{w}
$$

and more generally, by induction, we have

$$
\left|\left(\phi_{P^{i}} \phi_{R_{i}} \phi_{G}\right)(\beta)\right|_{w}=C_{w}|P|_{w}^{i},
$$

for any $i$. Thus, letting $f$ be the smallest $i$ such that $R_{i} \neq 0$, we have

$$
\left|\phi_{Q}(\beta)\right|_{w}=\left|\left(\phi_{R_{f} P^{f}} \phi_{G}\right)(\beta)\right|_{w}=C_{w}|P|_{w}^{f} .
$$

Since $f \leq \operatorname{ord}_{P}(Q)$, this completes our proof of Lemma 3.12 .

Using Lemma 3.12, we obtain

$$
\log \left|\phi_{Q}(\beta)\right|_{w} \geq \log \left(C_{w}\right)+\operatorname{deg}(Q) \log |P|_{w},
$$

which proves (3.9.1) and thus concludes the proof of Proposition 3.9.

The following corollary is an immedate consequence of Proposition 3.9 (see also Corollary 3.8). 
Corollary 3.13. Let $L$ be a finite extension of $K$ and let $\beta \in L$ be a nontorsion point. Then for every $w \in M_{L}$,

$$
\widehat{h}_{w}(\beta)=\lim _{\operatorname{deg}(Q) \rightarrow \infty} \frac{\log \left|\phi_{Q}(\beta)\right|_{w}}{\operatorname{deg}\left(\phi_{Q}\right)} .
$$

We are now ready to prove Theorem 3.1 .

Proof of Theorem 3.1. Using the product formula applied to the leading coefficient of $F$, we see that it suffices to prove our result under the assumption that $F$ is monic. Write $F=\prod_{i=1}^{n}\left(Z-\theta_{i}\right)$. Then

$$
\begin{aligned}
& \sum_{v \in M_{K}} \lim _{\operatorname{deg} Q \rightarrow \infty} \frac{1}{q^{d \operatorname{deg} Q}} \sum_{\phi_{Q}(y)=0} \log |F(y)|_{v} \\
& =\sum_{v \in M_{K}} \lim _{Q \rightarrow \infty} \frac{1}{q^{d \operatorname{deg} Q}} \sum_{\phi_{Q}(y)=0} \sum_{i=1}^{n} \log \left|y-\theta_{i}\right|_{v} \\
& =\sum_{v \in M_{K}} \lim _{Q \rightarrow \infty} \frac{1}{q^{d \operatorname{deg}(Q)}} \sum_{i=1}^{n} \log \left|\phi_{Q}\left(\theta_{i}\right)\right|_{v} \\
& \quad-\sum_{v \in M_{K}} \lim _{\operatorname{deg} Q \rightarrow \infty} \frac{n \log \left|\gamma_{Q}\right|_{v}}{q^{d \operatorname{deg} Q}}
\end{aligned}
$$

where $\gamma_{Q}$ is the leading coefficient of $\phi_{Q}$. Using induction, it is easy to see that $\log \left|\gamma_{Q}\right|_{v}=\frac{q^{d \operatorname{deg} Q}-1}{q^{d}-1} \log \left|c a_{d}\right|_{v}$, where $a_{d}$ is the leading coefficient of $\phi_{t}$ and $c$ is the leading coefficient of $Q$ (which is in $\mathbb{F}_{q}$ ). Passing to the limit, we then find that

$$
\lim _{\operatorname{deg} Q \rightarrow \infty} \frac{\log \left|\gamma_{Q}\right|_{v}}{q^{d \operatorname{deg} Q}}=\frac{\log \left|a_{d}\right|_{v}}{q^{d}-1} .
$$

Applying the product formula to $a_{d}$ we thus see that the sum

$$
\sum_{v \in M_{K}} \lim _{\operatorname{deg} Q \rightarrow \infty} \frac{\log \left|\gamma_{Q}\right|_{v}}{q^{d \operatorname{deg} Q}}
$$

vanishes. Hence, it will suffice to show that

$$
(\operatorname{deg} F)(\widehat{h}(\beta))=\sum_{v \in M_{K}} \lim _{\operatorname{deg} Q \rightarrow \infty} \sum_{i=1}^{n} \frac{\log \left|\phi_{Q}\left(\theta_{i}\right)\right|_{v}}{q^{d \operatorname{deg} Q}} .
$$

Now, let $L:=K(\beta)$, and let $p^{t}$ (for some $t \geq 0$ ) be the inseparable degree of $L / K$. Then $n=p^{t} s$, where $s$ is the separable degree of $L / K$. Moreover, the $\theta_{i}$ above (for $1 \leq i \leq n$ ) split into $s$ multisets, each of them consisting of $p^{t}$ identical elements (while different multisets have different elements). Therefore we may choose distinct $\theta_{1}, \ldots, \theta_{s}$ among the $\theta_{i}$ above. Let $K_{1}:=K^{1 / p^{t}}$, and let $L_{1}$ be the compositum (inside $\bar{K}$ ) of $K_{1}$ and $L$. Then $L_{1} / K_{1}$ is a separable extension of degree $s$. Moreover, the irreducible polynomial $F_{1}$ of $\beta \in L_{1}$ over $K_{1}$ equals $F^{1 / p^{t}}$. Dividing both sides of (3.13.2) by $p^{t}$, we obtain

$$
\left(\operatorname{deg} F_{1}\right)(\widehat{h}(\beta))=\sum_{v \in M_{K_{1}}} \lim _{\operatorname{deg} Q \rightarrow \infty} \sum_{i=1}^{s} \frac{\log \left|\phi_{Q}\left(\theta_{i}\right)\right|_{v}}{q^{d \operatorname{deg} Q}}
$$


Note that for (3.13.3) we used the fact that above each place $v \in M_{K}$ lies a unique place of $K_{1}$ (which we also denote by $v$ ). Therefore we are left to prove (3.13.2) in the case where $\beta$ generates a separable extension. Thus from now on we assume $L / K$ is a separable extension of degree $n$.

Following [Ser97, page 11], each absolute value of some $x \in L_{w}$ (for $w \mid v$ ) takes the form $\left|\mathrm{N}_{L_{w} / K_{v}}(x)\right|_{v}^{1 /[L: K]}$. Writing $\mathrm{N}_{L_{w} / K_{v}}(x)$ as $\iota_{w, 1}(x) \cdots \iota_{w, m_{w}}(x)$, where $\iota_{w, 1}, \ldots, \iota_{w, m_{w}}$ are the embeddings of $L_{w}$ into $\mathbb{C}_{v}$ (and $m_{w}=\left[L_{w}: K_{v}\right]$ ), we have

$$
|x|_{w}^{[L: K]}=\prod_{j=1}^{m_{w}}\left|\iota_{w, j}(x)\right|_{v} .
$$

For each $\theta_{i}$ in $\mathbb{C}_{v}$, there is exactly one pair $(w, j)$ such that $\iota_{w, j}(\beta)=\theta_{i}$. So, using the fact that $\phi_{Q}\left(\theta_{i}\right)=\iota_{w, j}\left(\phi_{Q}(\beta)\right)$ (because $\phi_{Q}$ has coefficients in $K$ ), we sum over all $L_{w}$ and obtain

$$
\sum_{i=1}^{n} \log \left|\phi_{Q}\left(\theta_{i}\right)\right|_{v}=[L: K] \sum_{w \mid v} \log \left|\phi_{Q}(\beta)\right|_{w} .
$$

Passing to the limit, and using the fact that $[L: K]=(\operatorname{deg} F)$, we thus obtain

$$
\begin{aligned}
\sum_{v \in M_{K}} \lim _{\operatorname{deg} Q \rightarrow \infty} \sum_{i=1}^{n} \frac{\log \left|\phi_{Q}\left(\theta_{i}\right)\right|_{v}}{q^{d \operatorname{deg} Q}} & =\sum_{w \in M_{L}} \lim _{Q \rightarrow \infty}(\operatorname{deg} F) \cdot \frac{\log \left|\phi_{Q}(\beta)\right|_{w}}{q^{d \operatorname{deg} Q}} \\
& =(\operatorname{deg} F) \sum_{w \in M_{L}} \widehat{h}_{w}(\beta) \\
& =(\operatorname{deg} F) \widehat{h}(\beta),
\end{aligned}
$$

as desired.

We can extend the conclusion of Theorem 3.1 and prove the following result.

Theorem 3.14. For any $\beta \in \bar{K}$ and any nonzero irreducible $F \in K[Z]$ such that $F(\beta)=0$, we have

$$
(\operatorname{deg} F) \widehat{h}(\beta)=\sum_{v \in M_{K}} \lim _{\operatorname{deg} Q \rightarrow \infty} \frac{1}{q^{d \operatorname{deg} Q}} \sum_{\substack{\phi_{Q}(y)=0 \\ F(y) \neq 0}} \log |F(y)|_{v} .
$$

Proof. The difference from Theorem 3.1 is that in Theorem 3.14 we also deal with torsion points $\beta$. This is why we exclude from the inner summation of (3.14.1) the case $F(y)=0$. Theorem 3.14 for $\beta \in \phi_{\text {tor }}$ follows exactly as Theorem 3.1 once we prove the following proposition.

Proposition 3.15. Assume $\beta \in \bar{K}$ is a torsion point. Then

$$
\sum_{v \in M_{K}} \lim _{\operatorname{deg} Q \rightarrow \infty} \frac{1}{q^{d \operatorname{deg} Q}} \sum_{\substack{\phi_{Q}(y)=0 \\ y \neq \beta}} \log |y-\beta|_{v}=0 .
$$

Proof of Proposition 3.15. Let $v \in M_{K}$. For each $Q \in A$ we let $\gamma_{Q}$ be the leading coefficient of $\phi_{Q}$. Also, let $a_{d}$ be the leading coefficient of $\phi_{t}$. We will prove that

$$
\lim _{\operatorname{deg} Q \rightarrow \infty} \frac{1}{q^{d \operatorname{deg} Q}} \sum_{\substack{\phi_{Q}(y)=0 \\ y \neq \beta}} \log |y-\beta|_{v}=-\frac{\log \left|a_{d}\right|_{v}}{q^{d}-1} .
$$


Using (3.15.1) and the product formula for $a_{d}$ we will then conclude the proof of Proposition 3.15

We proceed to proving (3.15.1). Let $Q \in A$ be a nonconstant polynomial. There are two cases:

Case 1. $\phi_{Q}(\beta)=0$.

Then

$$
\prod_{\substack{\phi_{Q}(y)=0 \\ y \neq \beta}}(\beta-y)=\frac{\left(\phi_{Q}\right)^{\prime}(\beta)}{\gamma_{Q}} .
$$

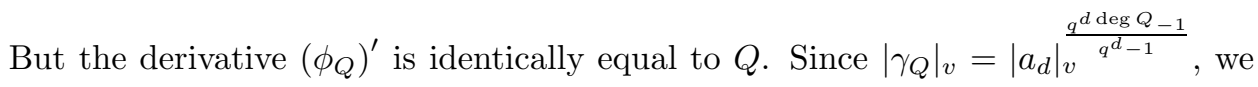
have

$$
\begin{aligned}
\lim _{\operatorname{deg} Q \rightarrow \infty} \frac{1}{q^{d \operatorname{deg} Q}} \sum_{\substack{\phi_{Q}(y)=0 \\
y \neq \beta}} \log |y-\beta|_{v} & =\lim _{\operatorname{deg} Q \rightarrow \infty} \frac{\log |Q|_{v}-\frac{q^{d \operatorname{deg} Q}-1}{q^{d}-1} \log \left|a_{d}\right|_{v}}{q^{d \operatorname{deg} Q}} \\
& =-\frac{\log \left|a_{d}\right|_{v}}{q^{d}-1},
\end{aligned}
$$

as desired.

Case 2. $\phi_{Q}(\beta) \neq 0$.

Then

$$
\prod_{\substack{\phi_{Q}(y)=0 \\ y \neq \beta}}(\beta-y)=\prod_{\phi_{Q}(y)=0}(\beta-y)=\frac{\phi_{Q}(\beta)}{\gamma_{Q}} .
$$

However, $\phi_{Q}(\beta)$ is one of the finitely many nonzero torsion points of $\phi$ of order dividing the order of $\beta$. Therefore, taking logarithms of (3.15.2) and using $\left|\gamma_{Q}\right|_{v}=$

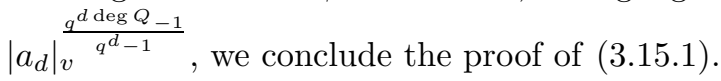

Arguing as in the proof of Theorem 3.1 we deduce the remaining case of Theorem 3.14 (i.e. $\beta \in \phi_{\text {tor }}$ ) from Proposition 3.15 .

We are now ready to prove Theorem 1.1 .

Proof of Theorem 1.1. Since $\beta$ is in $K$, the polynomial $F$ satisfied by $\beta$ in the statement of Theorem 3.1 may be taken to be $F(Z)=Z-\beta$. Let

$$
\phi_{t}(X)=a_{d} X^{q^{d}}+a_{d-1} X^{q^{d-1}}+\cdots+a_{1} X^{q}+t X .
$$

Then the leading coefficient $\gamma_{Q}$ of $\phi_{Q}$ equals $c a_{d}^{\frac{q^{d \operatorname{deg}(Q)}-1}{q^{d}-1}}$, where $c \in \mathbb{F}_{q}$ is the leading coefficient of $Q$. We observe that $\left|\gamma_{Q}\right|_{v} \neq 1$ if and only if $\left|a_{d}\right|_{v} \neq 1$. Then, after taking products followed by logarithms, we see that

$$
\sum_{\phi_{Q}(y)=0} \log |y-\beta|_{v}=\log \left|\phi_{Q}(\beta)\right|_{v}-\log \left|\gamma_{Q}\right|_{v} .
$$

Now, suppose that there are infinitely many $Q$ such that $\phi_{Q}(\beta)$ is $S$-integral for 0 . Let $\mathcal{T}$ be the set of all places in $S$ along with all the places $v$ for which $|\beta|_{v}>1$ and all the places of bad reduction for $\phi$. For any $Q$, at any $v$ outside of $\mathcal{T}$, we have $|y|_{v} \leq 1$ when $\phi_{Q}(y)=0$ (see Fact 2.3). Since $|\beta|_{v} \leq 1$, then $\log |y-\beta|_{v} \leq 0$ for $v \notin \mathcal{T}$. 
When $\phi_{Q}(\beta)$ is $S$-integral for 0 , it is certainly $\mathcal{T}$-integral as well. So, for all $v$ outside $\mathcal{T}$, we have $\sum_{y} \log |y-\beta|_{v} \geq 0$, by (3.15.4) (note that $\left|\gamma_{Q}\right|_{v}=1$ and $\left|\phi_{Q}(\beta)\right|_{v} \geq 1$ because $\phi_{Q}(\beta)$ is $\mathcal{T}$-integral). Hence, for these $Q$, we have $\log |y-\beta|_{v}=0$ for all $v$ outside $\mathcal{T}$, and for all $y$ such that $\phi_{Q}(y)=0$. Since $\mathcal{T}$ is finite, we can interchange the limit and the sum in (3.1.1) and apply the product formula, which yields $\widehat{h}(\beta)=0$. This is a contradiction, since $\beta$ is nontorsion.

Remark 3.16. It is worth noting that our alternative way of computing local heights (see Corollary 3.13) also holds for $\beta \in \mathbb{C}_{w}$ if $w$ does not lie over $v_{\infty}$. For example, our key Lemma 3.12 holds for every nontorsion point in $\mathbb{C}_{w}$, not only for algebraic points (i.e. for points in $\bar{K}$ ). However, Corollary 3.13 cannot be extended to all $\beta \in \mathbb{C}_{\infty}$ as shown by the following example. This is the case because Bosser's result (our Proposition 3.2) does not hold for linear forms in logarithms of transcendental points in $\mathbb{C}_{\infty}$ (i.e points not in $\bar{K}$ ).

Example 3.17. We construct an example of a point $\beta \in \mathbb{C}_{\infty}$ for which the limit $\lim _{\operatorname{deg} Q \rightarrow \infty} \frac{\log \left|\phi_{Q}(\beta)\right|_{\infty}}{q^{d \operatorname{deg} Q}}$ does not exist.

We work with the simplest Drinfeld module (but a similar construction works for any Drinfeld module as the reader will easily see). So, we let $\phi: \mathbb{F}_{q}[t] \rightarrow \mathbb{F}_{q}(t)\{\tau\}$ be the Carlitz module, i.e. $\phi_{t}(x)=t x+x^{q}$. By abuse of notation, we denote by $\infty$ the place $v_{\infty}$ of $\mathbb{F}_{q}(t)$. The Carlitz module has associated to it a 1-dimensional lattice at $\infty$ which is spanned by an element called $\zeta$, say.

Let $(d(n))_{n \geq 1}$ be a sequence of positive integers which is very rapidly increasing, i.e. $d(n+1)$ is much larger than $q^{d(n)}$. For the sake of concreteness, we will use the sequence $d(n)$ defined recursively, by letting $d(1):=q$ and for every $n \geq 1$, $d(n+1):=q^{q^{d(n)}}$.

Let $\alpha:=\zeta \cdot \sum_{n \geq 1} \frac{1}{t^{d(n)}} \in \mathbb{C}_{\infty}$. Clearly $\alpha$ is a (very rapidly) convergent power series.

Let $\beta:=\exp _{\phi}(\alpha)$, where $\exp _{\phi}$ is the usual exponential map of $\phi$ at $\infty$. This is the transcendental point (i.e. not in $\bar{K}$ ) which will give us our counterexample because $\left|\phi_{t^{d(n)}}(\beta)\right|_{\infty}$ will be much too rapidly decreasing.

Indeed, $\phi_{t^{d(n)}}(\beta)=\exp _{\phi}\left(t^{d(n)} \alpha\right)$, and using the fact that $\exp _{\phi}$ kills everything in $\mathbb{F}_{q}[t] \cdot \zeta$, we conclude that

$$
\phi_{t^{d(n)}}(\beta)=\exp _{\phi}\left(\zeta \cdot \sum_{m>n} \frac{1}{t^{d(m)-d(n)}}\right) .
$$

Now, because our sequence $(d(m))_{m \geq 1}$ was chosen to be rapidly increasing, $\left|\frac{1}{t^{d(n+1)-d(n)}}\right|_{\infty}$ is sufficiently small (for sufficiently large $n$ ) and so, $\zeta \cdot \sum_{m>n} \frac{1}{t^{d(m)-d(n)}}$ is in a sufficiently small ball around 0 on which $\exp _{\phi}$ preserves the metric (see Lemma 3.6). Moreover,

$$
\left|\zeta \cdot \sum_{m>n} \frac{1}{t^{d(m)-d(n)}}\right|_{\infty}=|\zeta|_{\infty} \cdot\left|\frac{1}{t^{d(n+1)-d(n)}}\right|_{\infty} .
$$

Therefore, we have

$$
\left|\phi_{t^{d(n)}}(\beta)\right|_{\infty}=|\zeta|_{\infty} \cdot\left|\frac{1}{t^{d(n+1)-d(n)}}\right|_{\infty}=|\zeta|_{\infty} \cdot e^{-(d(n+1)-d(n))},
$$


so $\log \left|\phi_{t^{d(n)}}(\beta)\right|_{\infty}=\log |\zeta|_{\infty}-(d(n+1)-d(n))$. Since $d(n+1)-d(n)$ is much larger than $q^{d(n)}$ (because $\left.d(n+1)=q^{q^{d(n)}}\right)$, we see that $\log \left|\phi_{t^{d(n)}}(\beta)\right|_{\infty}$ is much smaller than $-q^{d(n)}$. More precisely,

$$
\lim _{n \rightarrow \infty} \frac{d(n+1)-d(n)}{q^{d(n)}}=\lim _{n \rightarrow \infty} \frac{d(n+1)}{q^{d(n)}}=\lim _{n \rightarrow \infty} \frac{q^{q^{d(n)}}}{q^{d(n)}}=+\infty,
$$

showing that we do not have a finite limit for

$$
\frac{\log \left|\phi_{Q}(\beta)\right|_{\infty}}{q^{\operatorname{deg}(Q)}} \text { as } \operatorname{deg} Q \rightarrow \infty
$$

\section{An analog of a theorem of Schinzel}

Using our findings we are able to prove the following result, which is similar to a result of Schinzel [Sch74 for primitive divisors of $B^{n}-C^{n}$ in number fields. It came to our attention that independently, Hsia [Hsi] proved a similar Schinzel statement for Drinfeld modules. We thank him for pointing out the argument for our Lemma 4.2 .

Theorem 4.1. Let $K$ be a finite extension of $\mathbb{F}_{q}(t)$. Let $\beta \in K$ be a nontorsion point for $\phi$, and let $S$ be a finite set of places in $M_{K}$. Then there exists a positive integer $N$ such that for all $Q \in \mathbb{F}_{q}[t]$ of degree at least $N$, there exists $v \in M_{K} \backslash S$ such that

(i) $\left|\phi_{Q}(\beta)\right|_{v}<1$; and

(ii) for every nonzero polynomial $P \in \mathbb{F}_{q}[t]$ that divides $Q$ and has smaller degree than $Q$, we have $\left|\phi_{P}(\beta)\right|_{v} \geq 1$.

We begin by proving a precise result combining Lemmas 3.10 and 3.12 .

Lemma 4.2. Let $v \in M_{K}$ be a place lying above the irreducible polynomial $P \in$ $\mathbb{F}_{q}[t]$. Let $l:=\operatorname{deg} P$. For any $x \in K$, if

$$
|x|_{v}<|P|_{v}^{\frac{1}{q^{q^{-}-1}}}<1
$$

then for every $Q \in \mathbb{F}_{q}[t]$, we have $\left|\phi_{Q}(x)\right|_{v}=|Q x|_{v} \leq|x|_{v}$.

Proof. If $Q \in \mathbb{F}_{q}[t]$ is coprime with $P$, then $|Q|_{v}=1$. Thus $|x|_{v}<|Q|_{v}$ and so, using Lemma 3.10, we conclude that $\left|\phi_{Q}(x)\right|_{v}=|Q x|_{v}$.

Let $\phi_{P}=\sum_{i=0}^{d l} b_{i} \tau^{i}$. We know that $b_{0}=P$ (by the definition of $\phi$ ). If

$$
|x|_{v}<\min _{1 \leq i \leq d l}\left(\left|\frac{P}{b_{i}}\right|_{v}\right)^{\frac{1}{q^{i}-1}}
$$

then for every $1 \leq i \leq d l$, we have $|P x|_{v}>\left|b_{i} x^{q^{i}}\right|_{v}$; so, $\left|\phi_{P}(x)\right|_{v}=|P x|_{v}$. We will next show that $|x|_{v}<|P|_{v}^{\frac{1}{q^{l}-1}}$ implies (4.2.1). First we prove that $\left|b_{i}\right|_{v} \leq|P|_{v}$ for every $1 \leq i \leq l-1$.

If $l=1$, then the above claim is vacuously true. Therefore, assume $l>1$. We will use induction on $i$ to show that $\left|b_{i}\right|_{v} \leq|P|_{v}$ for every $1 \leq i \leq l-1$. Since $\phi_{P} \phi_{t}=\phi_{t} \phi_{P}$, writing $\phi_{t}=\sum_{i=0}^{d} a_{i} \tau^{i}$, and noting that the coefficient of $\tau$ is the same in $\phi_{t} \phi_{P}$ as it is in $\phi_{P} \phi_{t}$, we obtain

$$
a_{0} b_{1}+a_{1} b_{0}^{q}=b_{0} a_{1}+b_{1} a_{0}^{q} .
$$


Since $b_{0}=P$ and $a_{0}=t$, (4.2.2) gives rise to the equality

$$
\left|b_{1}\right|_{v} \cdot\left|t^{q}-t\right|_{v}=\left|a_{1}\right|_{v} \cdot\left|P^{q}-P\right|_{v} \leq 1 \cdot|P|_{v}=|P|_{v} .
$$

Since we assumed $l>1$, we have $\left|t^{q}-t\right|_{v}=1$. Hence, the equation above yields $\left|b_{1}\right|_{v} \leq|P|_{v}$, as desired. Proceeding by induction, we now assume that $\left|b_{j}\right|_{v} \leq|P|_{v}$ for all $1 \leq j<i$. Equating coefficients of $\tau^{i}$ in both $\phi_{t} \phi_{P}$ and $\phi_{P} \phi_{t}$, we obtain

$$
a_{0} b_{i}+\sum_{j>1} a_{j} b_{i-j}^{q^{j}}=b_{i} a_{0}^{q^{i}}+\sum_{j<i} b_{j} a_{i-j}^{q^{j}} .
$$

Since $\left|b_{j}\right|_{v} \leq|P|_{v}$ for all $1 \leq j<i$ by assumption (and $\left|a_{j}\right|_{v} \leq 1$ for every $j$ ), we see that $\left|b_{i}\right|_{v} \cdot\left|t^{q^{i}}-t\right|_{v} \leq|P|_{v}$. But $t^{q^{i}}-t$ is divisible only by irreducible polynomials in $\mathbb{F}_{q}[t]$ of degree at most $i$ (since $t^{q^{i}-1}-1$ splits completely over a degree $i$ extension of $\mathbb{F}_{q}$ ). Therefore, $\left|t^{q^{i}}-t\right|_{v}=1$ (because $i<l$ ), so $\left|b_{i}\right|_{v} \leq|P|_{v}$, as desired.

Since $\left|b_{i}\right|_{v} \leq|P|_{v}<1$ for every $1 \leq i \leq l-1$, and $\left|b_{i}\right|_{v} \leq 1$ for every $i \geq l$, we have

$$
|P|_{v}^{\frac{1}{q^{l}-1}} \leq \min _{1 \leq i \leq d l}\left(\left|\frac{P}{b_{i}}\right|_{v}\right)^{\frac{1}{q^{i}-1}} .
$$

So, if $|x|_{v}<|P|_{v}^{\frac{1}{q^{l}-1}}$, then (4.2.1) holds and we have $\left|\phi_{P}(x)\right|_{v}=|P x|_{v}$. Moreover, $|P x|_{v}=|P|_{v} \cdot|x|_{v}<|P|_{v}$ and so, using Lemma 3.10, we see that

$$
\left|\phi_{P^{2}}(x)\right|_{v}=\left|\phi_{P}\left(\phi_{P}(x)\right)\right|_{v}=\left|P \phi_{P}(x)\right|_{v}=\left|P^{2} x\right|_{v} .
$$

Similarly, an easy induction shows that for every $i \geq 0$, we have $\left|\phi_{P^{i}}(x)\right|_{v}=\left|P^{i} x\right|_{v}$. For any (nonzero) $Q \in \mathbb{F}_{q}[t]$, there exists $i \geq 0$ and $R \in \mathbb{F}_{q}[t]$ coprime with $P$ such that $Q=P^{i} R$. Using $\left|\phi_{R}(x)\right|_{v}=|x|_{v}$, we thus obtain

$$
\left|\phi_{Q}(x)\right|_{v}=\left|\phi_{P^{i}}\left(\phi_{R}(x)\right)\right|_{v}=\left|P^{i}\right|_{v} \cdot\left|\phi_{R}(x)\right|_{v}=|Q|_{v} \cdot|x|_{v},
$$

and our proof is complete.

Corollary 4.3. Let $x \in K$. For all but finitely many places $v \in M_{K}$, if $\pi_{v} \in K$ is an uniformizer at $v$, and $|x|_{v} \leq\left|\pi_{v}\right|_{v}$, then $\left|\phi_{Q}(x)\right|_{v}=|Q x|_{v}$ for every $Q \in \mathbb{F}_{q}[t]$. More precisely, if $|x|_{v}<1$ and $v$ lies over a place of $\mathbb{F}_{q}(t)$ corresponding to an irreducible polynomial of degree larger than $\log _{q}\left(\left[K: \mathbb{F}_{q}(t)\right]+1\right)$, then $\left|\phi_{Q}(x)\right|_{v}=$ $|Q x|_{v}$ for every $Q \in \mathbb{F}_{q}[t]$.

Proof. Let $v \in M_{K}$ lie over the irreducible polynomial $P \in \mathbb{F}_{q}[t]$, and let $l=\operatorname{deg} P$. By Lemma 4.2, if $|x|_{v}<|P|_{v}^{\frac{1}{q^{l}-1}}$, then $\left|\phi_{Q}(x)\right|_{v}=|Q x|_{v}$ for every $Q \in \mathbb{F}_{q}[t]$. But $|P|_{v} \geq\left|\pi_{v}\right|_{v}^{\left[K: \mathbb{F}_{q}(t)\right]}$. Therefore, if $q^{l}-1>\left[K: \mathbb{F}_{q}(t)\right]$, then

$$
|P|_{v}^{\frac{1}{q^{l}-1}}>\left|\pi_{v}\right|_{v}
$$

Thus, if $|x|_{v} \leq\left|\pi_{v}\right|_{v}$, then (by Lemma 4.2) $\left|\phi_{Q}(x)\right|_{v}=|Q x|_{v}$ for every $Q \in \mathbb{F}_{q}[t]$. Finally, note that there are finitely many polynomials in $\mathbb{F}_{q}[t]$ having bounded degree.

We will also need the following technical result about local heights for our proof of Theorem 4.1 
Lemma 4.4. Let $\beta \in K$ be a nontorsion point for the Drinfeld module $\phi$. Then there exist positive constants $C_{0}$ and $N_{0}$ (depending on $\phi$ and $\beta$ ) such that for every place $v \in M_{K}$ for which $\widehat{h}_{v}(\beta)>0$, and for every $Q \in A=\mathbb{F}_{q}[t]$ of degree larger than $N_{0}$, we have

$$
q^{d \operatorname{deg}(Q)} \widehat{h}_{v}(\beta)-C_{0} \leq \log \left|\phi_{Q}(\beta)\right|_{v} \leq q^{d \operatorname{deg}(Q)} \widehat{h}_{v}(\beta)+C_{0} .
$$

Proof. Because we allow the constants $C_{0}$ and $N_{0}$ from the conclusion of Lemma 4.4 to depend on $\beta$, it suffices to prove our lemma for each (of the finitely many places) $v \in M_{K}$ for which $\widehat{h}_{v}(\beta)>0$.

Let $\phi_{t}=\sum_{i=0}^{d} a_{i} \tau^{i}$. For a fixed place $v \in M_{K}$ for which $\widehat{h}_{v}(\beta)>0$, we define

$$
M_{v}:=\max \left\{\left(\frac{\left|a_{i}\right|_{v}}{\left|a_{d}\right|_{v}}\right)^{\frac{1}{q^{d}-q^{2}}}: 0 \leq i<d\right\} \cup\left\{\frac{1}{\left|a_{d}\right|_{v}^{\frac{1}{q^{d}-1}}}\right\} .
$$

Let $N_{0}$ be a positive integer such that $\left|\phi_{t^{N_{0}}}(\beta)\right|_{v}>M_{v}$ (we can find such $N_{0}$ because $\left(\left|\phi_{t^{n}}(\beta)\right|_{v}\right)_{n}$ is unbounded). Let $\gamma:=\left|\phi_{t^{N_{0}}}(\beta)\right|_{v}$. The definition of $M_{v}$ yields

$$
\left|\phi_{t^{N_{0}+1}}(\beta)\right|_{v}=\left|\phi_{t}\left(\phi_{t^{N_{0}}}(\beta)\right)\right|_{v}=\left|a_{d}\right|_{v} \cdot \gamma^{q^{d}}>\gamma>M_{v}
$$

A simple induction shows that for every $n \geq 1$, we have

$$
\left|\phi_{t^{N_{0}+n}}(\beta)\right|_{v}=\left|a_{d}\right|_{v^{\frac{q^{d n}-1}{d^{d}-1}}}^{q^{q^{n d}}} .
$$

Hence,

$$
\widehat{h}_{v}(\beta)=\lim _{n \rightarrow \infty} \frac{\log \left|\phi_{t^{N_{0}+n}}(\beta)\right|_{v}}{q^{d\left(N_{0}+n\right)}}=\frac{\log \left|a_{d}\right|_{v}}{q^{d N_{0}}\left(q^{d}-1\right)}+\frac{\log \gamma}{q^{d N_{0}}},
$$

which means that

$$
\log \left|\phi_{t^{N_{0}+n}}(\beta)\right|_{v}=q^{d\left(N_{0}+n\right)} \widehat{h}_{v}(\beta)-\frac{\log \left|a_{d}\right|_{v}}{q^{d}-1} .
$$

Moreover, for every $Q \in A$ of degree $N_{0}+n$ (for $n \geq 1$ ), we have

$$
\log \left|\phi_{Q}(\beta)\right|_{v}=\log \left|\phi_{t^{N_{0}+n}}(\beta)\right|_{v}=q^{d\left(N_{0}+n\right)} \widehat{h}_{v}(\beta)-\frac{\log \left|a_{d}\right|_{v}}{q^{d}-1}
$$

which concludes the proof of Lemma 4.4 .

The following lemma is the key to proving Theorem 4.1 ,

Lemma 4.5. With the hypothesis from Theorem 4.1 , let $Q \in \mathbb{F}_{q}[t]$ be a nonconstant monic polynomial. Let $\mathcal{T}$ be the set of places $v \in M_{K}$ that satisfy the following properties:

(i) if $P \in \mathbb{F}_{q}[t]$ is an irreducible polynomial for which $|P|_{v}\langle 1$, then $\operatorname{deg} P>$ $\log _{q}\left(\left[K: \mathbb{F}_{q}(t)\right]+1\right)$

(ii) $\beta$ is a v-adic integer;

(iii) $v$ is a place of good reduction for $\phi$;

(iv) either $\left|\phi_{Q}(\beta)\right|_{v}=1$, or there is some $P \neq Q$ in $\mathbb{F}_{q}[t]$ such that $P \mid Q$ and $\left|\phi_{P}(\beta)\right|_{v}<1$

(v) $v \notin S$.

Then

$$
\sum_{v \in \mathcal{T}} \sum_{y \text { has order } Q} \log |\beta-y|_{v} \geq-\operatorname{deg} Q
$$


Proof. Since

$$
\sum_{\substack{v \in M_{K} \\ v \text { does not lie over } v_{\infty}}} \log |Q|_{v}=-\log |Q|_{v_{\infty}}=-\operatorname{deg} Q
$$

and no place $v \in \mathcal{T}$ lies over $v_{\infty}$, it will suffice to show that for each $v \in \mathcal{T}$, we have

$$
\sum_{y \text { has order } Q} \log |\beta-y|_{v} \geq \log |Q|_{v} .
$$

If $\left|\phi_{Q}(\beta)\right|_{v}=1$, then for every $y$ such that $\phi_{Q}(y)=0$, we have $|y-\beta|_{v}=1$. Indeed, Fact 2.3 implies $|y|_{v} \leq 1$ for all torsion points $y$, because all $v \in \mathcal{T}$ are places of good reduction for $\phi$. Moreover, $|\beta-y|_{v} \leq 1$ for $v \in \mathcal{T}$ and $y \in \phi_{\text {tor }}$ due to (ii). Furthermore, the leading coefficient $\gamma_{Q}$ of $\phi_{Q}$ is a $v$-adic unit. Thus

$$
\log \left|\phi_{Q}(\beta)\right|_{v}=\sum_{\phi_{Q}(y)=0} \log |y-\beta|_{v} .
$$

Hence, if $\left|\phi_{Q}(\beta)\right|_{v}=1$, then indeed $|y-\beta|_{v}=1$ for all $y$ of order dividing $Q$. Therefore (4.5.2) holds in this case because $|Q|_{v} \leq 1$ for $v \in \mathcal{T}$.

Thus, we may assume that $\left|\phi_{Q}(\beta)\right|_{v}<1$. Let $P_{0}$ be the smallest degree monic polynomial dividing $Q$ such that $\left|\phi_{P_{0}}(\beta)\right|_{v}<1$. Then, since $|y-\beta|_{v} \leq 1$ for each torsion point $y$ and since the leading coefficient of $\phi_{P_{0}}$ is a $v$-adic unit, we have

$$
\sum_{\substack{\phi_{P}(y)=0 \\ P \mid Q \text { with } P \neq Q}} \log |\beta-y|_{v} \leq \sum_{\phi_{P_{0}}(y)=0} \log |\beta-y|_{v}=\log \left|\phi_{P_{0}}(\beta)\right|_{v} .
$$

Using again that the leading coefficient of $\phi_{Q}$ is a $v$-adic unit, we obtain

$$
\sum_{\phi_{Q}(y)=0} \log |\beta-y|_{v}=\log \left|\phi_{Q}(\beta)\right|_{v} .
$$

Since $v \in \mathcal{T}$ satisfies (i) and $\left|\phi_{P_{0}}(x)\right|_{v}<1$, we can use the more precise claim of Corollary 4.3 and derive

$$
\log \left|\phi_{Q}(\beta)\right|_{v}=\log \left|\frac{Q}{P_{0}}\right|_{v}+\log \left|\phi_{P_{0}}(\beta)\right|_{v} \geq \log |Q|_{v}+\log \left|\phi_{P_{0}}(\beta)\right|_{v} .
$$

Equations (4.5.3), (4.5.4), and (4.5.5) yield (4.5.2), which finishes the proof of Lemma 4.5

We define the Möbius function $\mu$ on the multiplicative set of all monic polynomials in $\mathbb{F}_{q}[t]$ by

$$
\begin{gathered}
\mu(1)=1, \\
\mu\left(Q_{1} Q_{2} \ldots Q_{n}\right)=(-1)^{n},
\end{gathered}
$$

if $Q_{1}, \ldots, Q_{n}$ are distinct irreducible, nonconstant polynomials, and

$$
\mu(f)=0 \text { if } f \text { is not squarefree. }
$$

Lemma 4.6. With the notation as in Lemma 4.5, for each $v \in \mathcal{T}$, we have

$$
\sum_{y \text { has order } Q} \log |y-\beta|_{v}=\sum_{P \mid Q} \mu\left(\frac{Q}{P}\right) \log \left|\phi_{P}(\beta)\right|_{v} .
$$


Proof. Since the leading coefficient of $\phi_{P}$ (for each nonzero polynomial $P$ ) is a $v$-adic unit for each place $v \in \mathcal{T}$, then for each $P \mid Q$, we have

$$
\sum_{\phi_{P}(y)=0} \log |y-\beta|_{v}=\log \left|\phi_{P}(\beta)\right|_{v} .
$$

Using (4.6.1) and the principle of inclusion and exclusion applied to the set of all $y$ such that $\phi_{Q}(y)=0$ (by counting them with respect to their corresponding orders $P \mid Q)$, we obtain the result of Lemma 4.6 .

We are now ready to prove Theorem 4.1 .

Proof of Theorem 4.1. After dividing $Q$ by its leading coefficient (which is a constant in $\mathbb{F}_{q}$ ), we may assume $Q$ is monic. We will argue by contradiction.

We begin by dividing the places of $K$ into three sets. We let $\mathfrak{S}$ denote the set of all places $v$ such that $\widehat{h}_{v}(\beta)>0$, let $\mathcal{T}$ be as in Lemma 4.5, and let $\mathcal{U}$ denote the remaining places. Note that $\mathfrak{S}$ and $\mathcal{U}$ are both finite, because $\mathcal{T}$ contains all but finitely many places in $M_{K}$ (here we are using the assumption that the conclusion of our Theorem 4.1 fails, because then condition (iv) in Lemma 4.5 is satisfied by all but finitely many places). Note that $S \subset \mathfrak{S} \cup \mathcal{U}$ because by assumption (v) from Lemma 4.5, the set $\mathcal{T} \cap S$ is empty.

We begin by dealing with the places in $\mathfrak{S}$. As proved in Lemma 4.4 there exists a positive integer $N_{0}$ and a positive constant $C_{0}$ such that for all $Q \in \mathbb{F}_{q}[t]$ of degree larger than $N_{0}$, and for all $v \in \mathfrak{S}$, we have

$$
q^{d \operatorname{deg}(Q)} \widehat{h}_{v}(\beta)-C_{0} \leq \log \left|\phi_{Q}(\beta)\right|_{v} \leq q^{d \operatorname{deg}(Q)} \widehat{h}_{v}(\beta)+C_{0} .
$$

Therefore, from now on, we will always assume $\operatorname{deg}(Q)>N_{0}$.

Next, we treat the places in $\mathcal{U}$. As proved in (3.7.2) and in Lemma 3.12, there exist positive constants $C_{1}, C_{2}$, and $C_{3}$ depending only on $\phi, \beta$ and $\mathcal{U}$ such that for all $v \in \mathcal{U}$,

$$
-C_{1} \operatorname{deg}(Q) \log \operatorname{deg}(Q)-C_{2}<\log \left|\phi_{Q}(\beta)\right|_{v}<C_{3} .
$$

The right hand side of (4.6.3) is guaranteed by the fact that $\widehat{h}_{v}(\beta)=0$ (because only $\mathfrak{S}$ contains places $v$ for which $\widehat{h}_{v}(\beta)>0$ ). Summing over all the $v$ in $\mathcal{U}$, we see that there are constants $C_{4}, C_{5}$, and $C_{6}$ such that

$$
-C_{4} \operatorname{deg}(Q) \log \operatorname{deg}(Q)-C_{5}<\sum_{v \in \mathcal{U}} \log \left|\phi_{Q}(\beta)\right|_{v}<C_{6} .
$$

Since there are finitely many polynomials $P$ of degree at most equal to $N_{0}$, we see from equations (4.6.2) and (4.6.4) that there are constants $C_{7}$ and $C_{8}$ such that

$$
\begin{aligned}
q^{d \operatorname{deg} P \widehat{h}(\beta)-C_{4} \operatorname{deg}(Q) \log \operatorname{deg}(Q)-C_{7}} & \leq \sum_{v \in \mathfrak{S} \cup \mathcal{U}} \log \left|\phi_{P}(\beta)\right|_{v} \\
& \leq q^{d \operatorname{deg} P \widehat{h}(\beta)+C_{8}}
\end{aligned}
$$

for all $P \mid Q$. Since $\mathcal{T}$ consists of all the places not in $\mathcal{U}$ or $\mathfrak{S}$, we thus obtain from the product formula that

$$
\begin{aligned}
q^{d \operatorname{deg} P \widehat{h}(\beta)-C_{4} \operatorname{deg}(Q) \log \operatorname{deg}(Q)-C_{7}} & \leq \sum_{v \in \mathcal{T}}-\log \left|\phi_{P}(\beta)\right|_{v} \\
& \leq q^{d \operatorname{deg} P \widehat{h}}(\beta)+C_{8}
\end{aligned}
$$


for all $P \mid Q$. Moreover, equation (4.6.1) gives

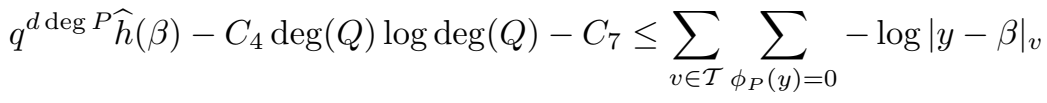

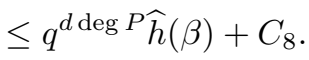

Using (4.6.7), we will bound

$$
\sum_{v \in \mathcal{T}} \sum_{\substack{\phi_{P}(y)=0 \\ \text { for } P \mid Q \text { with } P \neq Q}}-\log |\beta-y|_{v} .
$$

We compute the above sum via inclusion-exclusion (as we did in Lemma 4.6) and we obtain

$$
\sum_{\substack{P \mid Q \\ P \neq Q}}-\mu\left(\frac{Q}{P}\right) \sum_{v \in \mathcal{T}} \sum_{\phi_{P}(y)=0}-\log |\beta-y|_{v} .
$$

A simple computation using (4.6.7) and (4.6.8) shows that if $Q_{1}, \ldots, Q_{s}$ are all the distinct irreducible factors of $Q$, then

$$
\begin{aligned}
& \sum_{v \in \mathcal{T}} \sum_{\substack{\phi_{P}(y)=0 \\
\text { for } P \mid Q \text { with } P \neq Q}}-\log |\beta-y|_{v} \\
& \leq\left(q^{d \operatorname{deg}(Q)}-q^{d \operatorname{deg}(Q)} \prod_{i=1}^{s}\left(1-q^{-d \operatorname{deg}\left(Q_{i}\right)}\right)\right) \widehat{h}(\beta) \\
& \quad+2^{s}\left(C_{4} \operatorname{deg}(Q) \log \operatorname{deg}(Q)+C_{7}+C_{8}\right),
\end{aligned}
$$

due to the simple identity

$$
\sum_{\substack{P \mid Q \\ P \neq Q}}-\mu\left(\frac{Q}{P}\right) q^{d \operatorname{deg}(P)}=q^{d \operatorname{deg}(Q)}-q^{d \operatorname{deg}(Q)} \prod_{i=1}^{s}\left(1-q^{-d \operatorname{deg}\left(Q_{i}\right)}\right)
$$

and the fact that there are $2^{s}-1$ nonzero terms in the outer sum from (4.6.8). Using (4.6.7) for $P=Q$, we obtain

$$
\sum_{v \in \mathcal{T}} \sum_{\phi_{Q}(y)=0}-\log |y-\beta|_{v} \geq q^{d \operatorname{deg} Q} \widehat{h}(\beta)-C_{4} \operatorname{deg}(Q) \log \operatorname{deg}(Q)-C_{7} .
$$

But Lemma 4.5 and equation (4.6.9) imply that

$$
\begin{aligned}
& \sum_{v \in \mathcal{T}} \sum_{\phi_{Q}(y)=0}-\log |y-\beta|_{v} \\
& \leq \widehat{h}(\beta)\left(q^{d \operatorname{deg}(Q)}-q^{d \operatorname{deg}(Q)} \prod_{i=1}^{s}\left(1-q^{-d \operatorname{deg}\left(Q_{i}\right)}\right)\right) \\
& \quad+2^{s}\left(C_{4} \operatorname{deg}(Q) \log \operatorname{deg}(Q)+C_{7}+C_{8}\right)+\operatorname{deg} Q .
\end{aligned}
$$


Since $\widehat{h}(\beta)$ is positive, for $Q$ of large degree we have

$$
\begin{aligned}
& q^{d \operatorname{deg} Q} \widehat{h}(\beta)-C_{4} \operatorname{deg}(Q) \log (\operatorname{deg}(Q))-C_{7} \\
& >\widehat{h}(\beta)\left(q^{d \operatorname{deg}(Q)}-q^{d \operatorname{deg}(Q)} \prod_{i=1}^{s}\left(1-q^{-d \operatorname{deg}\left(Q_{i}\right)}\right)\right) \\
& \quad+2^{s}\left(C_{4} \operatorname{deg}(Q) \log \operatorname{deg}(Q)+C_{7}+C_{8}\right)+\operatorname{deg} Q
\end{aligned}
$$

because (recalling that $s$ is the number of distinct irreducible factors of $Q$, which is much smaller than $\operatorname{deg} Q$ as $\operatorname{deg} Q$ goes to infinity)

$$
\begin{aligned}
q^{d \operatorname{deg}(Q)} & \prod_{i=1}^{s}\left(1-q^{-d \operatorname{deg}\left(Q_{i}\right)}\right) \widehat{h}(\beta) \geq \sqrt{q^{d \operatorname{deg}(Q)}} \widehat{h}(\beta) \\
& >2^{s+1}\left(C_{8}+\operatorname{deg} Q+C_{4} \operatorname{deg}(Q) \log \operatorname{deg}(Q)+C_{7}\right) .
\end{aligned}
$$

Inequalities (4.6.10), (4.6.11) and (4.6.12) give us a contradiction, which means that there exists $v \in \mathcal{T}$ such that $\left|\phi_{Q}(\beta)\right|_{v}<1$ but $\left|\phi_{P}(\beta)\right|_{v}=1$ for all $P \mid Q$ with $P \neq Q$.

The following result is an immediate consequence of Theorem 4.1

Corollary 4.7. With the notation as in Theorem 4.1, there exists a positive integer $N$ such that for all monic $Q \in \mathbb{F}_{q}[t]$ of degree at least $N$, there exists a place $v \in M_{K}$ of good reduction for $\phi$, such that $\beta$ is integral at $v$ and $\bar{\beta}$ is a torsion point of order $Q$ for $\bar{\phi}$.

\section{FURTHER DIRECTIONS}

Let $\psi: \mathbb{P}^{1} \longrightarrow \mathbb{P}^{1}$ be a rational map of degree $d>1$ defined over a number field $L$. As with Drinfeld modules, one can define canonical heights $\widehat{h}_{v}$ for $\psi$, following Call and Goldstine ([CG97]). Piñeiro, Szpiro, and Tucker ([PST04] have proved that

$$
\widehat{h}_{v}(\beta)=\int_{\mathbb{P}^{1}\left(\mathbb{C}_{v}\right)} \log |F|_{v} d \mu_{v, \psi},
$$

where $\widehat{h}_{v}$ is the local canonical height for $\psi$ and $\mu_{v, \psi}$ is an invariant measure associated to $\psi$ on $\mathbb{P}^{1}\left(\mathbb{C}_{v}\right)$. This generalizes an earlier formula due to Mahler ( Mah60]). The invariant measure at an archimedean place $v$ was constructed by Lyubich ([Lyu83; see also Bro65] and [FLM83, Mañ88]), while the invariant measure at nonarchimedean places (which technically exists on the Berkovich space for $\mathbb{P}^{1}\left(\mathbb{C}_{v}\right)$, as defined in [Ber90]) was constructed by Baker/Rumely (BR06]), Chambert-Loir ([CL06]), and Favre/Rivera-Letelier ([FRL04, FRL]). These measures can also be constructed at places of a function field over a finite field (see Ghi06 for a treatment of these measures at places lying over $v_{\infty}$ ). Thus, Theorem 3.1 shows that a certain integral of the function $\log |x-\beta|_{v}$ may be computed by averaging this function over the torsion points of $\phi$. Hence, Theorem 3.1 is a statement about equidistribution. Note, however, that statements about equidistribution are usually phrased in terms of continuous functions (as is the case in Lyu83, BR06, CL06, FRL04, FRL, Ghi06. for example). Theorem 3.1 applies to a function that has a logarithmic pole at an algebraic number. 
Torsion points are merely inverse images of the point 0 . In light of the results of [ST] for rational functions over number fields, one is led to make the following conjecture.

Conjecture 5.1. Let $\alpha$ be any point in $\mathbb{G}_{a}(K)$. Then, for any nontorsion $\beta \in \bar{K}$ and any nonzero irreducible $F \in K[Z]$ such that $F(\beta)=0$, we have

$$
(\operatorname{deg} F) \widehat{h}(\beta)=\sum_{v \in M_{K}} \lim _{\operatorname{deg} Q \rightarrow \infty} \frac{1}{q^{d \operatorname{deg} Q}} \sum_{\phi_{Q}(y)=\alpha} \log |F(y)|_{v} .
$$

In BIR05, Baker, Ih, and Rumely show that local heights can also be computed by averaging $\log |x-\beta|_{v}$ over Galois orbits of torsion points of elliptic curves and of $\mathbb{G}_{m}$ over a number field. This is stronger, since the set of points $y$ such that $\psi^{m}(y)=e$ (where $e$ is the identity in the group) breaks into several Galois orbits of torsion points. The work of Baker, Ih, and Rumely leads us to conjecture the following.

Conjecture 5.2. Let $\left(\alpha_{n}\right)_{n=1}^{\infty}$ be any nonrepeating sequence of torsion points in $\mathbb{G}_{a}(\bar{K})$. Then for any nontorsion $\beta \in \bar{K}$ and any nonzero irreducible $F \in K[Z]$ such that $F(\beta)=0$, we have

$$
(\operatorname{deg} F) \widehat{h}(\beta)=\sum_{v \in M_{K}} \lim _{n \rightarrow \infty} \frac{1}{\#\left(\operatorname{Gal}(\bar{K} / K) \cdot \alpha_{n}\right)} \sum_{y \in \operatorname{Gal}(\bar{K} / K) \cdot \alpha_{n}} \log |F(y)|_{v} .
$$

Arguing as in the proof of Theorem 1.1. Conjecture 5.1 would yield the following corollary, which is analogous to a theorem proved by Silverman ( nonconstant morphisms of $\mathbb{P}^{1}$ of degree greater than one over a number field.

Corollary 5.3. Let $S$ be a finite set of places, let $\alpha$ be any point in $\mathbb{G}_{a}(K)$, and let $\beta$ be any nontorsion point in $\mathbb{G}_{a}(\bar{K})$. If Conjecture 5.1 holds, then there are finitely many $Q$ such that $\phi_{Q}(\beta)$ is $S$-integral for $\alpha$.

Similarly, Conjecture 5.2 would imply the following corollary, which was proved by Baker, Rumely, and Ih (BIR05) for elliptic curves and for $\mathbb{G}_{m}$ over a number field. Baker, Ih, and Rumely have conjectured that an analog of this holds for arbitrary nonconstant morphisms of degree greater than one of $\mathbb{P}^{1}$ over a number field.

Corollary 5.4. Let $S$ be a finite set of places. If Conjecture 5.2 holds, then for each nontorsion $\beta \in \mathbb{G}_{a}(\bar{K})$ there are finitely many torsion points $\alpha$ that are $S$-integral for $\beta$.

In the case of rational functions over number fields, the analog of Conjecture 5.1 has already been proved while the analog of Conjecture 5.2 is still just a conjecture (though some special cases have been proved, as noted earlier). In the case of Drinfeld modules, on the other hand, a proof of Conjecture 5.2 does not seem far off. Indeed, it would follow from a combination of the Tate-Voloch conjecture (proved in Ghi07b]) with suitable equidistribution results for continuous functions on the Berkovich space for $\mathbb{P}_{K}^{1}$. Such results have been proved in the number field case by Baker/Rumely ([BR06]), Chambert-Loir ([CL06]), and Favre/RiveraLetelier ([FRL04, FRL]). M. Baker informs us that the techniques used in these proofs should also work over function fields over finite fields.

It is less clear how one might proceed towards a proof of Conjecture 5.1. The arguments involving linear forms in logarithms at the infinite places work exactly 
the same, but the proof of Lemma 3.12 does not seem to work when 0 is replaced by an arbitrary point on $\mathbb{G}_{a}(\bar{K})$. Nevertheless, we are tempted to ask whether something even more general than Corollary 5.3 is true.

Conjecture 5.5. Let $S$ be a finite set of places, let $\alpha$ be any point in $\mathbb{G}_{a}(\bar{K})$, and let $\Gamma$ be any finitely generated $\phi$-submodule of $\mathbb{G}_{a}(\bar{K})$. Then there are finitely many $\gamma \in \Gamma$ such that $\gamma$ is $S$-integral for $\alpha$.

This would say the same thing for finitely generated $\phi$-submodules of $\mathbb{G}_{a}(\bar{K})$ that Siegel's theorem says for finitely generated $\mathbb{Z}$-submodules of $\mathbb{G}_{m}(\bar{K})$. Theorem 1.1 shows that our Conjecture 5.5 holds when $\Gamma$ is a cyclic $\phi$-submodule and $\alpha=0$ (note that is also a special case of Corollary 5.3). We can easily generalize this to a result that applies for any $\alpha \in \phi_{\text {tor }}(K)$ (when $\Gamma$ is a cyclic $\phi$-submodule), as we shall see next. We also mention that in [GT07] we proved Conjecture 5.5] under the assumption that $K$ has only one infinite place, and in addition assuming that Bosser's result on linear forms in logarithms holds also at finite places. While the second assumption is believed to be true by experts in the field, we also believe that the assumption from our paper [GT07] of having only one infinite place in $K$ could be removed, and so, Conjecture 5.5 would hold in the generality we stated here.

Following the same reasoning we used in deducing Theorem 1.1 from Theorem 3.1 (which in turn was deduced from Corollary 3.13), we can prove Conjecture 5.5 in the case $\alpha \in \phi_{\text {tor }}(K)$ and $\Gamma$ is a cyclic $\phi$-submodule generated by a nontorsion point $\beta$, from the following proposition.

Proposition 5.6. If $\beta$ is a nontorsion point, and $\alpha \in K$ is a torsion point, then for every place $w \in M_{K(\beta)}$, we have

$$
\widehat{h}_{w}(\beta)=\lim _{\operatorname{deg} Q \rightarrow \infty} \frac{\log \left|\phi_{Q}(\beta)-\alpha\right|_{w}}{q^{d \operatorname{deg} Q}} .
$$

We noted earlier that Corollary 5.3 would follow easily from Conjecture 5.1 via the arguments used in the derivation of Theorem 1.1 from Theorem 3.1. Now, reasoning as in the proof of Theorem 3.1, we see that Proposition 5.6 yields Conjecture 5.1 in the case where $\alpha$ is torsion because (after reducing to the case that $F(Z)=\prod_{i=1}^{n}\left(Z-\theta_{i}\right)$ is separable, as we did in the proof of Theorem 3.1) we have

$$
\sum_{i=1}^{n} \sum_{\phi_{Q}(y)=\alpha} \log \left|y-\theta_{i}\right|_{v}=n \cdot\left(\sum_{\substack{w \mid v \\ w \in M_{K(\beta)}}} \log \left|\phi_{Q}(\beta)-\alpha\right|_{w}\right)-n \log \left|\gamma_{Q}\right|_{v}
$$

for each $v \in M_{K}$ (where $\gamma_{Q}$ is as before the leading coefficient of $\phi_{Q}$ ). The above equality follows readily from Proposition 5.6 using the arguments that appeared in the proof of Theorem 3.1, after noting the bijection between the two sets

$$
\left\{y: \phi_{Q}(y)=\alpha\right\} \text { and }\left\{\alpha_{0}+y: \phi_{Q}(y)=0\right\} \text {, }
$$

where $\alpha_{0}$ is a fixed solution for $\phi_{Q}(x)=\alpha$. Therefore all we need to do is prove Proposition 5.6. We provide a sketch of a proof below.

Proof of Proposition 5.6. If $\beta$ is not in the filled Julia set for $\phi$ at $w$, then

$$
\left|\phi_{Q}(\beta)-\alpha\right|_{w}=\left|\phi_{Q}(\beta)\right|_{w}
$$


for polynomials $Q$ of large degree. Thus, in this case, the conclusion of Proposition 5.6 is immediate.

Assume from now on that $\beta$ is in the filled Julia set for $\phi$ at $w$. Then we need to show that

$$
\lim _{\operatorname{deg} Q \rightarrow \infty} \frac{\log \left|\phi_{Q}(\beta)-\alpha\right|_{w}}{q^{d \operatorname{deg} Q}}=0 .
$$

Let $\epsilon>0$. Since $\left|\phi_{Q}(\beta)\right|_{w}$ is bounded as $\operatorname{deg} Q \rightarrow \infty$, it follows that

$$
\limsup _{\operatorname{deg} Q \rightarrow \infty} \frac{\log \left|\phi_{Q}(\beta)-\alpha\right|_{w}}{q^{d \operatorname{deg} Q}}<\epsilon
$$

It will suffice to also show that

$$
\liminf _{\operatorname{deg} Q \rightarrow \infty} \frac{\log \left|\phi_{Q}(\beta)-\alpha\right|_{w}}{q^{d \operatorname{deg} Q}>-\epsilon}
$$

If $w$ lies over $v_{\infty}$, then an argument almost identical to the one used in Proposition 3.7 will give rise to (5.6.3). As with Proposition 3.7, the key ingredient is an application of the lower bound for linear forms in logarithms as provided by Proposition 3.2 .

If $w$ does not lie over $v_{\infty}$, then a slight modification of our argument from Proposition 3.9 may be used to prove (5.6.3). For this we use the following claim.

Claim 5.7. Let $w$ be a place that does not lie over $v_{\infty}$. Then there exists a positive constant $C_{w}$ such that for every $Q \in A$, we have $\left|\phi_{Q}(\beta)-\alpha\right|_{w} \geq C_{w}|Q|_{w}$.

Proof of Claim 5.7. Let $R \in A$ be a nonconstant polynomial such that $\phi_{R}(\alpha)=0$ (we recall that $\alpha \in \phi_{\text {tor }}$ ). If for every $Q \in A$, we have $\left|\phi_{Q}(\beta)-\alpha\right|_{w} \geq|R|_{w}$, then the conclusion of Claim 5.7 is immediate. Therefore, assume there exists $Q \in A$ such that $\left|\phi_{Q}(\beta)-\alpha\right|_{w}<|R|_{w}$. For each such $Q$, Lemma 3.10 yields $\left|\phi_{R}\left(\phi_{Q}(\beta)-\alpha\right)\right|_{w}=|R|_{w} \cdot\left|\phi_{Q}(\beta)-\alpha\right|_{w}$, which implies that

$$
\left|\phi_{R Q}(\beta)\right|_{w}=|R|_{w} \cdot\left|\phi_{Q}(\beta)-\alpha\right|_{w}
$$

since $\phi_{R}(\alpha)=0$. By Lemma 3.12 there exists $C_{w}>0$ such that for every polynomial $Q^{\prime}$, we have $\left|\phi_{Q^{\prime}}(\beta)\right|_{w} \geq C_{w}\left|Q^{\prime}\right|_{w}$. Hence, in particular, we have $\left|\phi_{R Q}(\beta)\right|_{w} \geq C_{w} \cdot|R Q|_{w}$. Applying (5.7.1), we obtain $\left|\phi_{Q}(\beta)-\alpha\right|_{w} \geq C_{w}|Q|_{w}$, as desired.

Since $\lim _{\operatorname{deg} Q \rightarrow \infty} \frac{\log |Q|_{w}}{q^{d \operatorname{deg} Q}}=0$, Proposition [5.6 now follows immediately from Claim 5.7 .

\section{ACKNOWLEDGMENTS}

We would like to thank M. Baker and V. Bosser for many interesting conversations.

\section{REFERENCES}

[Ber90] V. G. Berkovich, Spectral theory and analytic geometry over nonarchimedean fields, AMS Mathematical Surveys and Monographs, American Mathematical Society, Providence, 1990. MR1070709 (91k:32038)

[BIR05] M. Baker, S. I. Ih, and R. Rumely, A finiteness property of torsion points, 2005, preprint, available at arxiv:math.NT/0509485, 30 pages.

[Bos99] V. Bosser, Minorations de formes linéaires de logarithmes pour les modules de Drinfeld, J. Number Theory 75 (1999), no. 2, 279-323. MR1681633 (2000b:11090) 
[Bos00] - Transcendance et approximation diophantienne sur les modules de Drinfeld, Ph.D. thesis, Université Paris 6, 2000.

[Bos02] Hauteurs normalisées des sous-variétés de produits de modules de Drinfeld, Compositio Math. 133 (2002), no. 3, 323-353. MR1930981 (2003g:11062)

[BR06] M. Baker and R. Rumely, Equidistribution of small points, rational dynamics, and potential theory, Ann. Inst. Fourier (Grenoble) 56 (2006), 625-688. MR2244226

[Bro65] H. Brolin, Invariant sets under iteration of rational functions, Ark. Mat. 6 (1965), 103-144. MR0194595 (33:2805)

[CG97] G. S. Call and S. Goldstine, Canonical heights on projective space, J. Number Theory 63 (1997), 211-243. MR:1443758 (98c:11060)

[CL06] A. Chambert-Loir, Mesures et équidistribution sur les espaces de Berkovich, J. Reine. Angew. Math 595 (2006), 215-235. MR2244803

[Den92a] L. Denis, Géométrie diophantienne sur les modules de Drinfeld, The arithmetic of function fields (Columbus, OH, 1991), Ohio State Univ. Math. Res. Inst. Publ., vol. 2, de Gruyter, Berlin, 1992, pp. 285-302. MR1196525 (93j:11033)

[Den92b] _ Hauteurs canoniques et modules de Drinfeld, Math. Ann. 294 (1992), no. 2, 213-223. MR 1183402 (94d:11038)

[FLM83] A. Freire, A. Lopes, and R. Mañe, An invariant measure for rational functions, Boletim da Sociedade Brasileira de Matematica 14 (1983), 45-62. MR736568 (85m:58110b)

[FRL] C. Favre and J. Rivera-Letelier, Équidistribution des points de petite hauteur, to appear in Math. Ann. MR2221116 (2007g:11074)

[FRL04] _ Théorème d'équidistribution de Brolin en dynamique p-adique, C. R. Math. Acad. Sci. Paris 339 (2004), no. 4, 271-276. MR2092012 (2005f:37090)

[Ghi05] D. Ghioca, The Mordell-Lang theorem for Drinfeld modules, Int. Math. Res. Not. (2005), no. 53, 3273-3307.

[Ghi06] D. Ghioca, Equidistribution for torsion points of a Drinfeld module, Math. Ann. 336 (2006), no. 4, 841-865. MR2255176 (2007g:11063)

[Ghi07a] _ The local Lehmer inequality for Drinfeld modules, J. Number Theory 123 (2007), no. 2, 426-455. MR2301224

[Ghi07b] _ The Tate-Voloch conjecture for Drinfeld modules, J. Number Theory 125 (2007), no. 1, 85-94. MR2333120

[Gos96] D. Goss, Basic structures of function field arithmetic, Ergebnisse der Mathematik und ihrer Grenzgebiete (3) [Results in Mathematics and Related Areas (3)], vol. 35, SpringerVerlag, Berlin, 1996. MR.1423131 (97i:11062)

[GT07] D. Ghioca and T. J. Tucker, Siegel's theorem for Drinfeld modules, Math. Ann. 339 (2007), no. 1, 37-60. MR2317762

[Hsi] L.-C. Hsia, On the reduction of a non-torsion point of a Drinfeld module, preprint.

[Lyu83] M. Lyubich, Entropy properties of rational endomorphisms of the Riemann sphere, Ergodic Theory Dynam. Systems 3 (1983), 351-385. MR741393 (85k:58049)

[Mah60] K. Mahler, An application of Jensen's formula to polynomials, Mathematica 7 (1960), 98-100. MR0124467 (23:A1779)

[Mañ88] R. Mañe, The Hausdorff dimension of invariant probabilities of rational maps, Dynamical Systems, Valparaiso 1986 (R. Bamon, R. Labarca, and J. Palis, eds.), SpringerVerlag, 1988, pp. 86-117. MR961095 (90j:58073)

[Osg75] C. F. Osgood, Effective bounds on the "Diophantine approximation" of algebraic functions over fields of arbitrary characteristic and applications to differential equations, Nederl. Akad. Wetensch. Proc. Ser. A 78=Indag. Math. 37 (1975), 105-119. MR0387204 (52:8048a)

[Pin04] R. Pink, On the order of the reduction of a point on an abelian variety, Math. Ann. 330 (2004), no. 2, 275-291. MR2089426 (2005g:11101)

[Poo95] B. Poonen, Local height functions and the Mordell-Weil theorem for Drinfeld modules, Compositio Math. 97 (1995), no. 3, 349-368. MR1353279 (96k:11075)

[PST04] J. Piñeiro, L. Szpiro, and T. Tucker, Mahler measure for dynamical systems on $\mathbb{P}^{1}$ and intersection theory on a singular arithmetic surface, Geometric methods in algebra and number theory (F. Bogomolov and Y. Tschinkel, eds.), Progress in Mathematics 235, Birkhäuser, 2004, pp. 219-250. MR2166086 (2006f:14026)

[Rot55] K. F. Roth, Rational approximations to algebraic numbers, Mathematika 2 (1955), 1-20, corrigendum, ibid. 2 (1955), 168. MR0072182(17:242d) 
[Sca02] T. Scanlon, Diophantine geometry of the torsion of a Drinfeld module, J. Number Theory 97 (2002), no. 1, 10-25. MR.1939133 (2003h:11063)

[Sch74] A. Schinzel, Primitive divisors of the expression $a^{n}-b^{n}$ in algebraic number fields, J. Reine Angew. 268/269 (1974), 27-33, Collection of articles dedicated to Helmut Hasse on his seventy-fifth birthday, II. MR0344221 (49:8961)

[Ser97] J.-P. Serre, Lectures on the Mordell-Weil theorem, third ed., Aspects of Mathematics, Friedr. Vieweg \& Sohn, Braunschweig, 1997, Translated from the French and edited by Martin Brown from notes by Michel Waldschmidt, with a foreword by Brown and Serre. MR.1757192(2000m:11049)

[Sie29] C. L. Siegel, Über einige anwendungen diophantisher approximationen, Abh. Preuss. Akad. Wiss. Phys. Math. Kl. (1929), 41-69.

[Sil93] J. H. Silverman, Integer points, Diophantine approximation, and iteration of rational maps, Duke Math. J. 71 (1993), no. 3, 793-829. MR1240603 (95e:11070)

[ST] L. Szpiro and T. J. Tucker, Equidistribution and generalized Mahler measures, preprint, Available at arxiv.org/abs/math. NT/0603438, 29 pages.

[SUZ97] L. Szpiro, E. Ullmo, and S. Zhang, Equirépartition des petits points, Invent. Math. 127 (1997), 337-347. MR:1427622 (98i:14027)

[Tag93] Y. Taguchi, Semi-simplicity of the Galois representations attached to Drinfeld modules over fields of "infinite characteristics", J. Number Theory 44 (1993), no. 3, 292-314. MR.1233291 (94k:11064)

[Vol90] J. F. Voloch, Explicit p-descent for elliptic curves in characteristic p, Compos. Math. (1990), no. 74, 247-258. MR1055695 (91f:11042)

[Vol91] On the conjectures of Mordell and Lang in positive characteristic, Invent. Math. (1991), no. 104, 643-646. MR1106753 (92d:11067)

[Vol97] Diophantine geometry in characteristic p: a survey, Arithmetic geometry (Cortona, 1994), Sympos. Math., 37, Cambridge Univ. Press, Cambridge, 1997, pp. 260-278. MR:1472501 (99c:11081)

Department of Mathematics, McMaster University, 1280 Main Street West, Hamilton, Ontario, Canada L8S 4K1

E-mail address: dghioca@math.mcmaster.ca

Current address: Department of Mathematics and Computer Science, University of Lethbridge, Lethbridge, Alberta, Canada T1K 3M4

E-mail address: dragos.ghioca@uleth.ca

Department of Mathematics, Hylan Building, University of Rochester, Rochester, NEW YORK 14627

E-mail address: ttucker@math.rochester.edu 